\title{
The Protective Effect of Anise, Insulin, and their Combination in the Nephropathy of Diabetic Rat Model
}

\author{
Original \\ Article \\ Rasha Ragab Abdo Selima, Moustafa Mahmoud El.Habiby, Abeer El-Said El- \\ Mehi and Manar Ali Faried
}

Department of Human Anatomy and Embryology, Faculty of Medicine, Menoufia University

\begin{abstract}
Introduction: Among the diabetic complications, the diabetic nephropathy (DN) is the commonest reason for end-stage kidney failure all over the world, and a major indication for dialysis and transplantation. Despite the number of studies on humans and rodents of experimental DN, successful treatment isn't accessible however So, the explore for drugs to prevent the progression of DN has a high priority in biomedical research

Aim: To examine the effect of anise on a diabetic nephropathy rat model by streptozotocin and compare its effect with insulin and with their combination.

Materials and Methods: Fifty-six adult male albino rats were divided into six main groups. Group I (control), Group II: received aniseed at a dose of $500 \mathrm{mg} / \mathrm{kg}$, Group III (diabetic): received a single IP of $50 \mathrm{mg} / \mathrm{kg}$ STZ, Group IV: diabetic rats received aniseed for eight weeks (as group II), Group V: received SC of $2 \mathrm{U} / \mathrm{Kg}$ once daily of an insulin mixture for eight weeks and Group VI: received aniseed and insulin as the previous doses for eight weeks. At the end of the study, blood samples were collected and the kidney specimens were processed biochemical, histological (light and transmission electron microscopic) and immunohistochemical studies. Morphometric and statistical studies were also performed.

Results: Diabetic rats showed disturbed renal architecture involving the corpuscles and tubules, a significant increase in collagen fibers, glomerulosclerosis index, and desmin, VEGF, keratin 18immunoreaction. Administration of aniseed showed impressive improvement of histological structural changes induced by diabetes, insulin showed a mild improvement and use of these agents in combination appeared to have potential advantages especially in reducing of oxidative stress in improving DN

Conclusions: A combination of aniseed and insulin have a relevant therapeutic role in the kidney changes induced by diabetes So, they could potentially provide a possible solution for renal changes related to diabetes
\end{abstract}

Received: 04 August 2020, Accepted: 30 August 2020

Key Words: Anise, diabetic nephropathy, Insulin, rat.

Corresponding Author: Rasha Ragab Abdo Selima, MSc, Department of Human Anatomy and Embryology, Faculty of Medicine, Menoufia University, Egypt, Tel.: +20 1065107689, E-mail: r.selima87@gmail.com

ISSN: 1110-0559, Vol. 44, No.2

\section{INTRODUCTION}

Diabetes mellitus (DM) is an important and chronic public health problem nowadays. It is characterized by high blood glucose resulting from a decrease in insulin secretion, impaired insulin action, or both ${ }^{[1]}$. Un controlled hyperglycemia is advance related with long term harm, failure and disappointment of numerous organs ${ }^{[2]}$. It is well known that failure of treatment of DM results in chronic complications including nephropathy, neuropathy and cardiomyopathy ${ }^{[3]}$. World Health Organization mentioned that about 366 million people will be diagnosed as diabetic at 2030. complications of the disease lead to an increase in death and morbidity rates ${ }^{[4]}$. Diabetic nephropathy is a disease that accompanied with renal damage, renal inflammation, and fibrosis ${ }^{[4]}$. Glomerular damage is observed as changes in renal structure, such as glomerulosclerosis, podocyte injury, mesangial matrix stretching, and tubuleinterstitial fibrosis ${ }^{[5]}$. It is a very important strategy to fight against diabetic complications is to prevent or delay the disease ${ }^{[6]}$.
Numerous herbs have been utilized to treat numerous kidney disorders, and few ponders have conducted in this scope. In between the traditional plants, there was Pimpinella anisum L. (anise), which was utilized at the treatment of renal stones ${ }^{[7]}$. P. anisum L. contains a characteristic fragrant odor having a place to the family of Apiaceae and local to the Mediterranean region, Western Asia, and the Middle East. It is broadly utilized in relief of digestive system related framework disarranges such as epigastria, moderate absorption, and flatulence ${ }^{[7]}$. The anti-diabetic effect of anise is attributed to its scavenging activity against nitric oxide and superoxide radicals; preventing hyperglycemia by modifying the activity of some key enzymes concerned in carbohydrate metabolism ${ }^{[8]}$. Moreover, few thinks about have highlighted the valuable effect of aniseed on kidney structure and its work; particularly against diabetic nephropathy. This work aimed to evaluate the effects of the Pimpinella anisum, insulin and their coadministration on the kidney of a rat model of diabetes mellitus by histological, immunohistochemical and biochemical studies. 


\section{MATERIALS AND METHODS}

\section{A- Chemicals}

- Streptozotocin (STZ) was available in a powder form $(1 \mathrm{gm})$ stored at- $20^{\circ} \mathrm{C}$ and was dissolved in distilled water. It was a product of Sigma-Aldrich Chemical Co., St. Louis. Mo, USA

- Insulin was purchased from Sigma-Aldrich (Munich, Germany) in the form of vials

- $\quad$ Anise extract: plant material

The seeds of P. anisum were obtained from a neighborhood herbs advertise and were identified and confirmed by a botanist at the Botany Department, Faculty of Science, Menoufia University

\section{B-Pimpinella anisum preparation}

The dried seeds of P. anisum were grounded into a powder. An amount of $500 \mathrm{~g}$ of this powder was poured into a clean cloth sack and after that set in a Soxhlet extraction swell. $70 \%$ Ethanol $(300 \mathrm{~mL})$ was poured into the Soxhlet carafe and the coming about blend was cleared out to stand for a day to get all of the extracts. Then, the extract was clarified using a Buchner Whatman filter paper. To get a concentrated extract, the alcohol dissolvable was expelled from the extract employing a rotating evaporator at a temperature $40^{\circ} \mathrm{C}$ of a water bath and a pressure of $275 \mathrm{mmHg}$. The coming about concentrated extract was expelled from the carafe and exchanged to a desiccator to dry ${ }^{[8]}$.

\section{c-Animals}

Fifty- six adult male albino rats, their average weight ranging from 150 to 200 grams, were utilized in this experiment. The rats were obtained and the study was done in Theodor Bilharz institute animal house, El-Warraq, Giza, Egypt. Upon entry, they were kept exclusively amid the total study in separated cages at room temperature beneath pathogen-free conditions to keep them in great wellbeing conditions with free access to a standard food and water.

\section{D-Experimental design}

Rats were kept for one week before the beginning of the experiment for acclimatization. At that point they were arbitrarily divided into six groups based on their body weight to accomplish around the same mean body weight in all groups.

Group I (Control): consisted of 16 male albino rats; they were subdivided into Subgroup Ia: consisted of 8 rats; they were kept without any treatment until the end of the study. Subgroup Ib: consisted of 4 rats, they received intraperitoneal (I.P) injection of $0.2 \mathrm{ml}$ of distilled water, the solvent of STZ at a once. Subgroup Ic: consisted of 4 rats, they received orally by gastric tube $0.2 \mathrm{ml}$ of distilled water, the solvent of aniseed at a once

Group II (aniseed): consisted of 8 rats, they received aniseed at a dose of $500 \mathrm{mg} / \mathrm{kg}$ dissolved in distilled water once daily for eight weeks orally by gastric tube ${ }^{[8]}$.
Group III (diabetic): consisted of 8 rats, received STZ once as a single at a dose of $50 \mathrm{mg} / \mathrm{kg}$ I.P dissolved in distilled water ${ }^{[9]}$. To confirm diabetes, three days after induction, a random blood glucose level was measured from the retro-orbital veins using one-touch glucose meter and strips (LifeScan, Inc. USA). Animals were considered diabetic with a blood glucose level of $\geq 250 \mathrm{mg} / \mathrm{dl}^{[10]}$.

Group IV (diabetic aniseed extract-treated): consisted of 8 diabetic rats; received $P$. anisum extract once daily with the previous dose of group II for eight weeks

Group V (diabetic insulin-treated): consisted of 8 diabetic rats; received a subcutaneous injection of $2 \mathrm{U} / \mathrm{Kg}$ once daily of an insulin mixture at $8 \mathrm{a} . \mathrm{m}$. for eight weeks ${ }^{[11]}$.

Group VI: (diabetic-aniseed extract \& Insulintreated): consisted of 8 diabetic rats. received insulin and aniseed extract with the previous doses for eight weeks.

\section{E-Calculation of the bodyweight}

The rats in each group were weighed at the end of the study and the mean body weight was calculated.

\section{F-Blood glucose level analysis}

The blood samples were obtained from retro-orbital veins and measured the blood glucose level by one-touch glucose meter

\section{G-Sample collection}

At the end of the study, rats were sacrificed by cervical dislocation. the kidney was dissected out. The left kidney was used for histopathological evaluation. The right kidney was used for biochemical assessment and a homogenate was prepared for further biochemical assessment

\section{H-Biochemical study}

Estimation of Glycosylated hemoglobin (HbA1c), plasma urea, and creatinine for detection of renal function using ELISA kits.

To obtain tissue homogenate, renal tissue was homogenized using a glass homogenizer in cold $0.9 \%$ $\mathrm{NaCl}$. For determination of the enzymatic activity of superoxide dismutase (SOD), Malondialdehyde (MDA), and Angiotensin-converting enzyme (ACE-1), tissue homogenates were centrifuged then the supernatant was used. All enzymatic assays were estimated at room temperature ${ }^{[12]}$.

\section{I-Histological study}

\section{I-Preparation of paraffin sections}

The left kidney specimens were fixed in $10 \%$ neutral formaldehyde, dehydrated, cleared and embedded in paraffin. Serial $5 \mu \mathrm{m}$ sections of the specimens were stained with Hematoxylin and Eosin, Masson`s trichrome stain and Periodic Acid Schiff (PAS) staining

Histological damage of the renal tubule was assessed as the percentage of the tubules that appeared dilated, atrophied, tubules with epithelial cell necrosis, and with cast formation 
as : 0 (normal); $1(<10 \%) ; 2$ ( 10 to $25 \%$ ); 3 (26 to $50 \%$ ); 4 (51 to $75 \%$ ) and $5(>75 \%)$. H\&E stained kidney segments were utilized to examine the renal tubular damage score. At a magnification of 200x, 10 regions of renal tubules were haphazardly chosen per kidney for the evaluation and an average score was calculated ${ }^{[13]}$.

PAS-stained paraffin sections were used to examine the degree of sclerosis inside the basement membrane of glomerular capillaries and the brush border of the PCTs by light microscopy at a magnification of $\mathrm{x} 400$, utilizing the semi-quantitative scoring framework: grade 0, normal glomeruli; grade 1 (minimal sclerosis) sclerosis up to 25 $\%$; grade 2, (moderate sclerosis) 25-50 \% sclerosis; grade 3, (moderate-severe sclerosis) 50-75\%sclerosis; grade 4, (very severe sclerosis) $75-100 \%$ sclerosis and then the glomerular score was determined. The glomerulosclerotic index (GSI) was calculated utilizing this equation: GSI $=$ $(1 \times \mathrm{n} 1)+(2 \times \mathrm{n} 2)+(3 \times \mathrm{n} 3)+(4 \times \mathrm{n} 4) / \mathrm{n} 0+\mathrm{n} 1+\mathrm{n} 2+\mathrm{n} 3$ $+\mathrm{n} 4$, where $\mathrm{n}$ is the number of glomeruli in each grade of glomerulosclerosis $^{[14]}$.

\section{II-Preparation of Transmission Electron Microscope (TEM) study}

Small pieces $(1 \mathrm{~mm} 3)$ from the kidney were cut and fixed immediately in a $2.5 \%$ glutaraldehyde solution, followed by $1 \%$ osmium tetroxide, got dried out, and then inserted in epoxy resin. Ultrathin sections were collected on copper grids and stained with uranyl acetate and lead citrate ${ }^{[12]}$. The ultrastructural examination was detected by a transmission electron magnifying lens JEOL JSM35 at an acceleration voltage of $20 \mathrm{kV}$ at the EM unit at the faculty of medicine, Tanta University.

\section{III - Immunohistochemical stains}

For immunohistochemistry, $5 \mu \mathrm{m}$-thick renal sections were deparaffinized, rehydrated, and washed in phosphatebuffered saline (PBS). At that point, the sections were hatched overnight at $4{ }^{\circ} \mathrm{C}$ in a humid chamber with the following primary antibodies; (rabbit polyclonal anti-rat antibody against desmin marker for podocyte injury, 1:50 dilution, Abcam), (rabbit polyclonal antibody against VEGF marker for angiogenesis, 1:200 dilution, Abcam), (rabbit polyclonal antibody against keratin 18 marker for epithelial cell injury, 1:100 dilution, segma) in PBS. Thereafter, it was washed in PBS buffer and co-incubated with a biotinylated secondary antibody for an hour at room temperature.

\section{IV-Morphometric measurements}

For histological and immunohistochemical quantitative assessment, the sections from at least five animals/ experimental group were examined. Five non-overlapping fields per section were randomly captured by a Lieca Microscope DML B2/11888111 equipped with a Leica camera DFC450. Kidney sections were randomly selected for morphometric measurements by using image analyzer software (Image J analyzer version 1.4308, National Institutes of Health, USA).
The following parameters were measured

1. The diameter of the renal corpuscle was measured X400

2. The area percentage of collagen fibers stained by Masson trichrome stain X200

3. The area percentage of (Desmin, VEGF \& Keratin 18) positive immune-staining $X 400$

\section{J-Statistical analysis}

All data were collected, revised, and subjected to statistical study using (ANOVA) test, and (Kruskal Wallis) test is used in the scoring of the tubular injury performed with SPSS.21 program and post-Hoc least significant difference. $P$-values $>0.05$ were considered non-significant and $P$-values $\leq 0.05$ were considered significant. The data were expressed as mean \pm standard deviation (SD) or as median and range

\section{RESULTS}

\section{Mortality rate}

No deaths were recorded in all groups.

\section{Bodyweight}

At the end of the experiment, there was a significant decrease $(p<0.001)$ in the bodyweight in the diabetic group in comparison with the control .But, the diabetic groups treated either with aniseed, insulin or aniseed \& insulin showed a significant increase $(p<0.01)$ in their weight in comparison with the diabetic group. Moreover, the diabetic aniseed treated showed a significant decrease $(p<0.01)$ in their weight compared to the diabetic insulin-treated. The combined treatment group showed a significant increase $(p<0.001)$ in its weight compared to the diabetic aniseed treated. Also, the combined treatment group showed a significant increase $(p<0.01)$ in its weight in comparison with the insulin-treated one (Table 1).

\section{Biochemical results}

1. Blood glucose levels: the diabetic group showed a significant increase $(p<0.001)$ in comparison with the control one. Meanwhile, there was a significant decrease in the diabetic groups treated either with aniseed, insulin, or aniseed \& insulin compared with the diabetic group. Moreover, the diabetic aniseed treated showed a significant increase $(p<0.01)$ in comparison with the insulin-treated one. The combined treatment group showed a significant decrease $(p<0.001)$ compared to the diabetic aniseed treated. Also, the combined treatment showed a significant decrease $(p<0.01)$ in comparison with insulin-treated one (Table 1)

2. Glycosylated hemoglobin $(\mathrm{HbAlc})$ : the diabetic group showed a significant increase $(p<0.001)$ in comparison with the control. Meanwhile, there was a significant decrease in the diabetic groups treated either with aniseed, insulin, or aniseed \& insulin 
compared with the diabetic untreated. Moreover, the diabetic aniseed treated showed a significant increase $(p<0.01)$ in comparison with the diabetic insulin-treated. The combined treatment showed a significant decrease $(p<0.001)$ compared to the diabetic aniseed treated. Also, the combined treatment group showed a significant decrease $(p<0.01)$ in comparison with the insulin-treated one (Table 1)

3. Plasma urea \&creatinine levels: the diabetic group showed a significant increase $(p<0.001)$ compared to the control group. The diabetic groups treated either with aniseed, insulin or aniseed \& insulin showed a significant decrease $(p<0.01)$ compared to the diabetic group. Moreover, the aniseed treated showed a significant decrease $(p<0.01)$ in comparison with the diabetic insulin-treated. The combined treatment group showed a significant decrease $(p<0.001)$ compared to the diabetic aniseed treated. Also, the combined treatment group showed a significant decrease $(p<0.01)$ compared to the insulin-treated (Table 2)

4. Superoxide dismutase (SOD), Malondialdehyde (MDA), and ACE-1 levels: the diabetic group showed a significant decrease $(p<0.001)$ in its SOD and ACE-1 with a significant increase $(p<0.001)$ in its MDA levels in comparison with the control. The diabetic groups treated either with aniseed, insulin or aniseed \& insulin showed a significant decrease $(p<0.01)$ in their MDA levels and an increase in their SOD and ACE-1 in comparison with the diabetic untreated. Meanwhile, the diabetic aniseed treated showed a significant increase $(p<0.01)$ in its SOD and ACE-1 with a significant decrease in its MDA in comparison with the insulin-treated one. The combined treatment showed a significant decrease $(p<0.001)$ in their MDA and an increase in SOD and ACE-1 levels in comparison with the aniseed treated. Also, the combined treatment group showed a significant decrease $(p<0.01)$ in their MDA and increase in SOD and ACE-1 levels compared to the diabetic insulin-treated (Table 3)

\section{Histological results}

In the present work, there wasn't any significant difference among group I (control group) and group II (aniseed group) in all examined parameters, so both groups were pooled in one group (control).

\section{A) Light microscopic results}

\section{$H$ and $E$ stain}

Histological examination of $\mathrm{H}$ and $\mathrm{E}$ stained sections of group I (control) showed the kidney had: an outer cortex lied beneath the renal capsule and an inner medulla that consisted of collecting tubules. The renal cortex is formed of renal corpuscles and renal tubules with different sizes. The renal corpuscles were roughly spherical with an inner globular lobulated capillary tuft which projected into the Bowmen's capsule that had two layers; the outer parietal layer and the inner visceral one. The capsular space separated the two layers from each other. The proximal convoluted tubules (PCTs) were with a narrow lumen. The distal convoluted ones (DCTs) appeared with a wider smooth lumen. The PCTs were lined with cuboidal epithelium with acidophilic cytoplasm and rounded vesicular nuclei but the DCTs were lined with columnar epithelium with less acidophilic cytoplasm and rounded vesicular nuclei. Meanwhile, the examination of the diabetic group showed distorted architecture of the renal cortex with shrinkage of their glomerular capillary tuft and widening of the Bowman's space with no lesions detected in the renal medulla. The PCTs and DCTs showed vacuolated cytoplasm, desquamation of their lining epithelium, and pyknotic nuclei.

Extravasation of the RBCs among tubules and peritubular inflammatory cell infiltration were also seen. Also, hypertrophied glomeruli with reduction of their Bowman's spaces, homogeneous cytoplasm of the surrounding renal tubules mostly hyalinization were detected). Also, large areas of cast formation and thick wall dilated congested blood vessel and excess inflammatory cell infiltration are seen (Figures 1a-e).

Examination of diabetic-aniseed treated showed restoration of normal renal architecture. The renal corpuscles and the renal tubules appeared nearly normal, scanty inflammatory cell infiltration can be seen and some renal tubules showed vacuolations and desquamation of their lining epithelium. Examination of diabetic- insulin-treated showed mild improvement in renal architecture. Normal glomerulus except for widening of the Bowman's space in some glomeruli, vacuolation of the renal tubular cells, and pyknosis of their nuclei could also be seen. Examination of diabetic- aniseed plus insulin-treated showed the renal architecture nearly similar to the control. The glomeruli and the renal tubules appeared nearly normal except for slight vacuolations and few cast formation (Figure 2a-c). Statistically, the diabetic group showed a significant decrease $(P<0.01)$ in renal corpuscles diameter and a significant increase in the tubular injury score compared to the control group, the diabetic groups treated either with aniseed, insulin or aniseed $\&$ insulin showed a significant increase $(p<0.01)$ in renal corpuscles diameter and a significant decrease in the tubular injury score compared to the diabetic group. Also, the diabetic aniseed treated and combined treatment groups showed a significant increase and significant decrease in the tubular injury score $(p<0.01)$ compared to the diabetic insulintreated. There was a non-significant difference $(p>0.05)$ in the diameter of the renal corpuscle and significant decrease in the tubular injury score $(p<0.01)$ between combined and aniseed treated groups (Table 4, Graphs 1,2)

\section{Masson's trichrome stain}

kidney sections of the control and combined treatment groups showed few collagen fiber depositions. The diabetic 
group showed an extremely large amount of collagen fiber deposition in the renal corpuscles, in the parietal layer of Bowman's capsule, and in between the renal tubules. Diabetic-aniseed treated showed a minimal amount of collagen fiber and diabetic-insulin-treated showed moderate collagen fiber deposition. Statistically, compared to the control, the diabetic group showed an extremely significant increase $(p<0.01)$ in its area percentage of collagen fibers. The diabetic groups treated either with aniseed or insulin or combined treatment showed a significant decrease $(p<0.01)$ in collagen fiber area percentage in comparison with the diabetic one. The diabetic aniseed treated and combined treatment groups showed a significant decrease $(p<0.01)$ compared to the diabetic insulin-treated. The combined treatment group showed a significant decrease $(p<0.01)$ in its collagen fiber deposition compared to the diabetic aniseed treated (Figure 3a-f), (Table 4).

\section{PAS stain}

Kidney sections of the control and combined treatment groups showed positive PAS reaction of the mesangium, the tubular basement membrane, and the glomerular capillary basement membrane as well as the brush border of the PCT cells but the diabetic group showed strong positive PAS reaction and thickening basement membranes in the Bowman's capsule. Diabetic-aniseed treated showed mild positive PAS reaction and diabetic-insulin-treated showed moderate to strong positive PAS reaction, Statistically, comparing with the control group, the diabetic group showed a significant increase $(p<0.01)$ in the glomerulosclerosis index (GSI). The diabetic groups treated either with aniseed or insulin or combined treatment showed a significant decrease $(p<0.01)$ in GSI in comparison with the diabetic untreated. The diabetic aniseed treated and combined treatment groups showed a significant decrease $(p<0.01)$ in GSI in comparison with the diabetic insulin-treated. The combined treatment group showed a significant decrease $(p<0.01)$ in GSI than that obtained by treatment with aniseed alone (Figure 4a-f), (Table 4)

\section{Transmission electron microscopic results}

Ultrastructure examination of the renal cortex of group I (control) showed the podocytes that appeared as large stellate cells which had numerous cytoplasmic extensions called primary processes that wrapped around the glomerular capillaries and secondary foot processes called pedicles that completely invested the glomerular capillaries. Also, there were narrow clefts in between the pedicles called filtration slits. The diabetic group showed podocytes wrapping around the congested glomerular capillary. Podocyte nuclei were hyperchromatic with peripheral condensation of chromatin, others appeared shrunken and pyknotic with a fusion of its secondary foot processes. The thickening of the glomerular capillary basal lamina could be noticed. The diabetic aniseed treated showed more or less normal podocytes with partial thickening of the capillary basement membrane. The diabetic insulin-treated group showed the podocyte with a hyperchromatic nucleus, diminution of their primary processes, and fusion of secondary foot processes. The aniseed plus insulin treated group showed that the architecture of the renal glomeruli and the renal tubules was greatly improved. The podocyte appeared as a large stellate cell with a normal nucleus and giving rise to numerous secondary foot processes (Figure 5a-f).

Ultrastructure examination of the PCTs of group I (control) showed a large oval euchromatic nucleus with peripheral heterochromatic nucleolus and homogenous cytoplasm. Long closely packed apical microvilli projecting into the lumen forming a prominent brush border, Pinocytotic vesicles were found near the apical surface. The diabetic group showed the PCTs with an abnormally shrunken nucleus and irregular nuclear envelope with large sized lysosomes. The diabetic aniseed treated group showed the PCTs appeared normal with few lysosomes. The diabetic insulin-treated group showed the PCTs with an irregular hyperchromatic nucleus, large-sized lysosomes, vacuolations, and wide intercellular space could be noticed with normal mitochondria but others degenerated. The aniseed plus insulin-treated group showed nearly normal PCTs except for some medium to large-sized lysosomes (Figure 6a-e).

Ultrastructure examination of the DCTs of group I (control) showed an apical oval to round euchromatic nucleus and the mitochondria appeared elongated and rounded with no microvilli. The diabetic group showed the DCTs with peripheral condensation of its chromatin, destructed mitochondria with rarefied cytoplasm. The diabetic aniseed treated group showed the DCTs with an apical oval euchromatic nucleus, regular nuclear envelop and mild chromatolysis except massive mitochondrial lysis could be noticed. The diabetic insulin-treated group showed the DCTs with euchromatic nuclei, some mitochondria appeared more or less normal but others showed loss of mitochondrial cisterns and wide intercellular space. The aniseed plus insulin-treated group showed the DCTs appeared with normal euchromatic rounded to oval nuclei and mitochondria of two shapes (elongated and rounded) appeared normal (Figure 7a-e).

\section{C-Immunohistochemical results}

\section{Desmin immunostaining}

Fine positive immunoreaction to desmin within the glomerulus was observed in the control and aniseed plus insulin-treated groups. The diabetic group showed overexpression of desmin stain within the glomerulus. While the diabetic aniseed treated showed mild immunoreaction to desmin within the glomerulus and insulin diabetic treated showed moderate immunoreaction to desmin within the glomerulus, Statistically, the diabetic group showed a significant increase $(p<0.01)$ in desmin area \% from the control group and the treated groups shows a significant decrease $(p<0.01)$ in the same percentage in comparison with the diabetic untreated. The diabetic aniseed treated and combined treatment groups showed a significant decrease $(p<0.01)$ in the same percentage $\%$ in comparison with the insulin-treated one. The combined treatment group 
showed a significant decrease $(p<0.001)$ in its desmin area $\%$ in comparison with the aniseed treated one (Figure 8a-f). (Table 5).

\section{VEGF immunostaining}

Slight cytoplasmic expression of VEGF in some renal tubular cells and glomerulus was observed in the control and aniseed plus insulin-treated groups. The diabetic group showed severe cytoplasmic expression of VEGF in the renal tubular cells and glomerulus. While the diabetic aniseed treated showed mild cytoplasmic expression of VEGF in the renal tubular cells and glomerulus and insulin diabetic treated showed moderate cytoplasmic expression. Statistically, the diabetic group showed a significant increase $(p<0.01)$ for VEGF area \% immunoreaction from the control group and the treated groups showed a significant decrease $(p<0.01)$ in VEGF area \% from the diabetic untreated. The diabetic aniseed treated and combined treatment groups showed a significant decrease $(p<0.01)$ in their VEGF area $\%$ in comparison with the insulin-treated group. The combined treatment showed a significant decrease $(p<0.001)$ in its VEGF area $\%$ in comparison with the aniseed treated one (Figure 9 a-f). (Table 5).

\section{Keratin 18 immunostaining}

In the control group, positive immunoreaction of keratin 18 was noticed in the tubules and the parietal cells of Bowman's capsule. In the diabetic group, overexpression of keratin 18 in many tubules and the parietal cells of Bowman's capsule could be seen. The diabetic aniseed treated revealed mild positive expression of keratin 18 and the diabetic insulin-treated showed moderate expression of keratin 18 in the tubules and the parietal cells of Bowman's capsule. While aniseed plus insulin-treated group revealed slight keratin 18 immunoreaction. Statistically, the diabetic group showed a significant increase $(p<0.01)$ in keratin 18 area $\%$ from the control, and the treated groups showed a significant decrease $(p<0.01)$ in keratin 18 area $\%$ from the diabetic untreated. The diabetic aniseed treated and combined treatment groups showed a significant decrease $(p<0.01)$ in their keratin 18 area $\%$ immunoreaction in comparison with the insulintreated. The combined treatment group showed a significant decrease $(\mathrm{p}<0.001)$ in its keratin 18 area \% in comparison with the aniseed treated one (Figure 10 a-f). (Table 5).

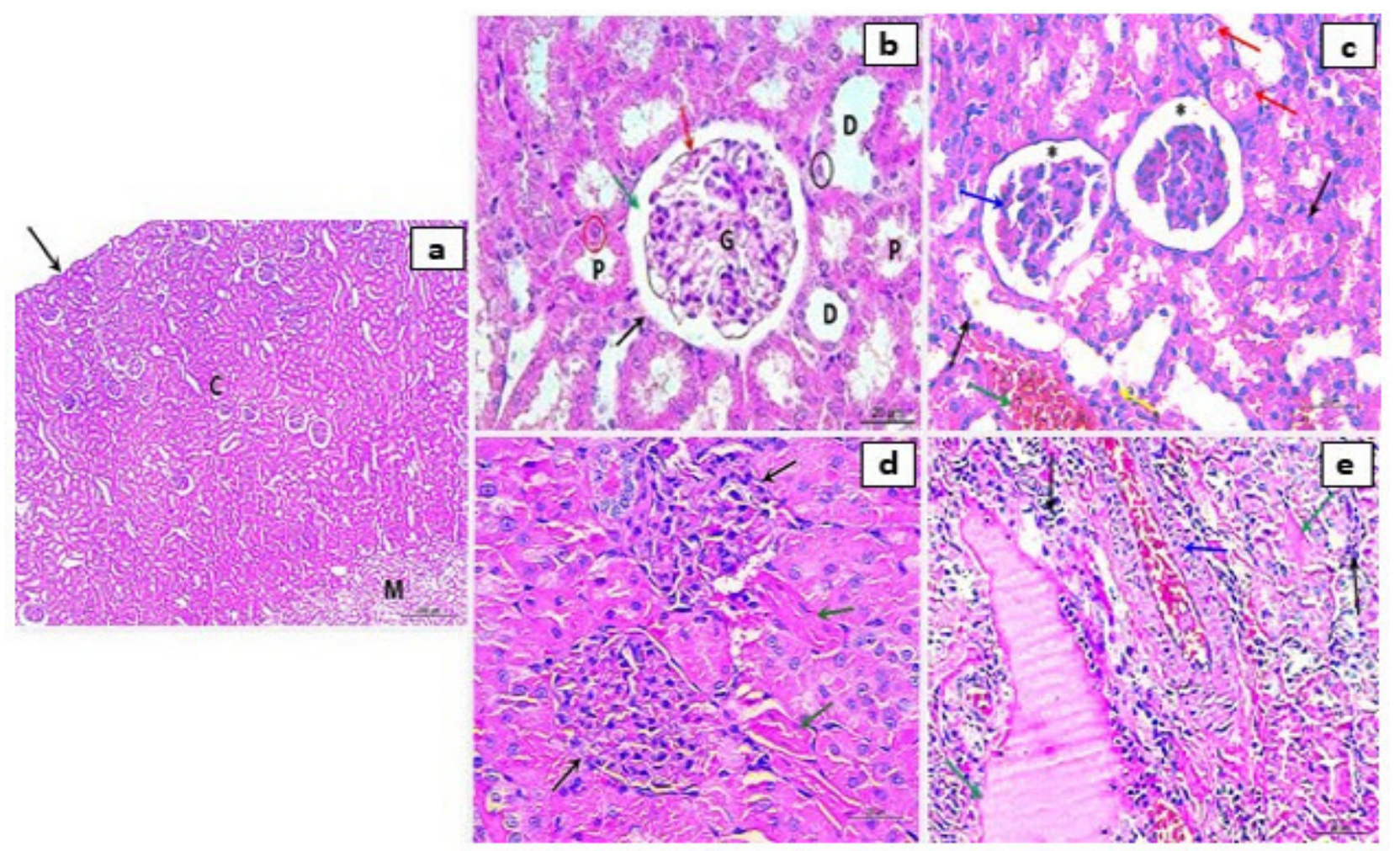

Fig. 1: Representative photomicrographs of the kidney from the group I (control) [a,b]:[a]the kidney is formed of an outer cortex(C) that lies beneath the renal capsule (black arrow) and inner medulla (M). [b] a renal corpuscle consists of a glomerulus (G) which projects into the Bowman's capsule that has two layers, the outer parietal layer (black arrow), inner visceral one (red arrow) separated by a capsular space (green arrow). The PCTs (P) are lined with cuboidal epithelium (red circle) with acidophilic cytoplasm. The DCTs (D) are lined with columnar epithelium (black circle). Group III (diabetic) [c,d,e]: [c] distorted glomerulus (blue arrow), with the widening of its Bowman's space (asterisks) can be noticed. The lining epithelial cells of the renal tubules showing vacuolated cytoplasm (red arrows), desquamation (yellow arrow), pyknotic nuclei (black arrows) and extravasation of RBCs among tubules (green arrow). [d] complete obliteration of the Bowman's spaces (black arrows) and homogenous cytoplasm of the surrounding renal tubules mostly hyalinization (green arrows) can be noticed. [e] large areas of cast formation (green arrows), thick wall dilated congested blood vessel (blue arrow) and excess inflammatory cell infiltration (black arrows) can be noticed. H and E [a X 40, Scale bar: $200 \mu \mathrm{m}$ ] [b,c,d,e X 400, Scale bar: $20 \mu \mathrm{m}$ ] 


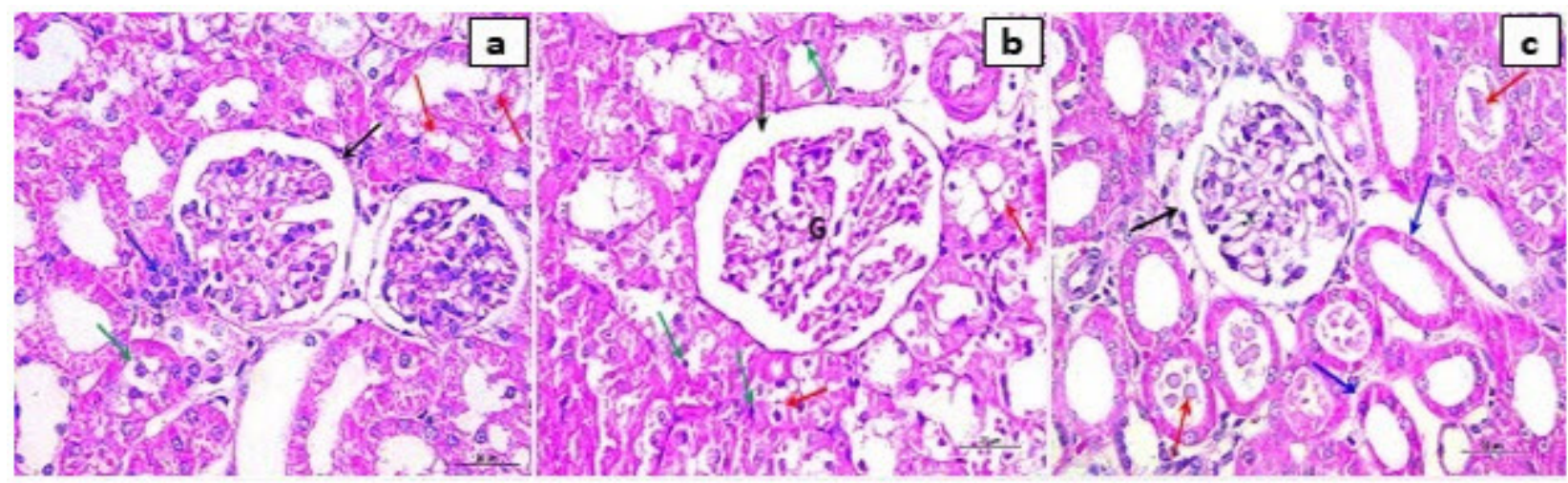

Fig. 2: Representative photomicrographs of the kidney from group IV (aniseed treated): [a] The renal corpuscles appear nearly normal (black arrow). Scanty inflammatory cell infiltration (blue arrow) and some renal tubules showing vacuolations (red arrows) or desquamation of their lining epithelium (green arrow). Group V (insulin-treated): [b] Normal glomerulus (G) except for a slight widening of the Bowman's space (black arrow) with vacuolation of the renal tubules (red arrows) and their nuclei showing pyknosis (green arrows). Group VI (combined treated): [c] The renal corpuscle (black arrow) and most of the renal tubules (blue arrows) appear normal except for cast formation (red arrows) in few tubules. H and E [ a,b,c X 400, Scale bar: $20 \mu \mathrm{m}]$

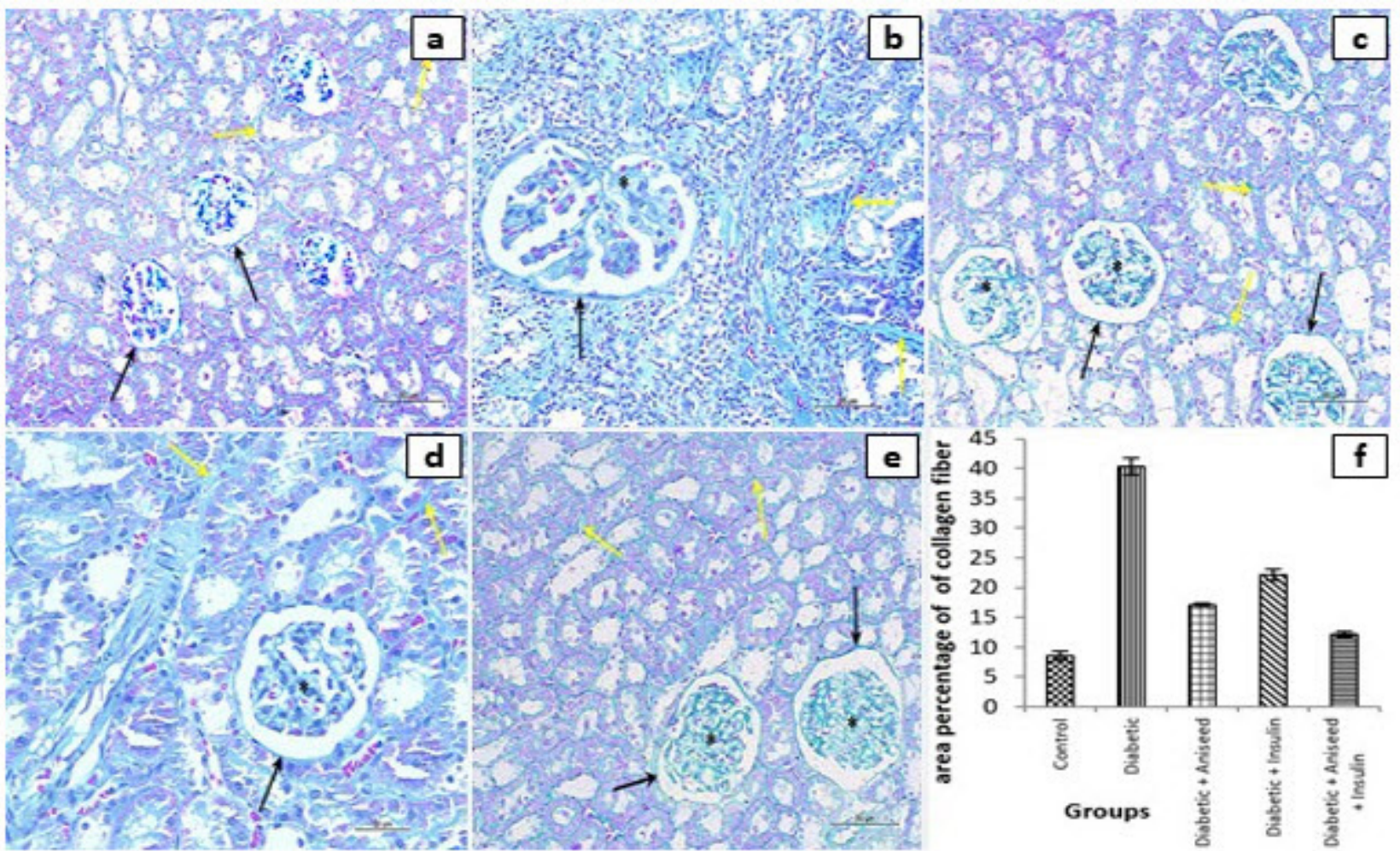

Fig. 3: Representative photomicrographs of the kidney from the group I (control) [a] fine collagen fiber deposition in the parietal layer of the Bowman's capsule (black arrows) and in between the renal tubules (yellow arrows) can be noticed. Group III [b] extremely large amount of collagen fiber deposition within the glomerulus (asterisk), in the parietal layer of the Bowman's capsule (black arrow) and in between the renal tubules (yellow arrows) can be noticed. Group IV(aniseed treated) [c] fine collagen fiber in between the renal tubules (yellow arrows) and in the parietal layer of the Bowman's capsule (black arrows). Some collagen fiber within the glomeruli are observed (asterisks) can be noticed. Group V (insulin-treated) [d] some collagen fiber in between the renal tubules (yellow arrows), within the glomerulus (asterisk) and in the parietal layer of the Bowman's capsule (black arrow) can be noticed. Group VI (combined treated) [e] fine collagen fiber in between the renal tubules (yellow arrows) and in the parietal layer of the Bowman's capsule (black arrows) nearly similar to the control group but moderate collagen deposition appears in the glomeruli (asterisks) can be noticed. [f]Graph of area percentage of collagen fiber deposition. Masson trichrome [a,b,c,d X 200, Scale bar: $50 \mu \mathrm{m}]$ 

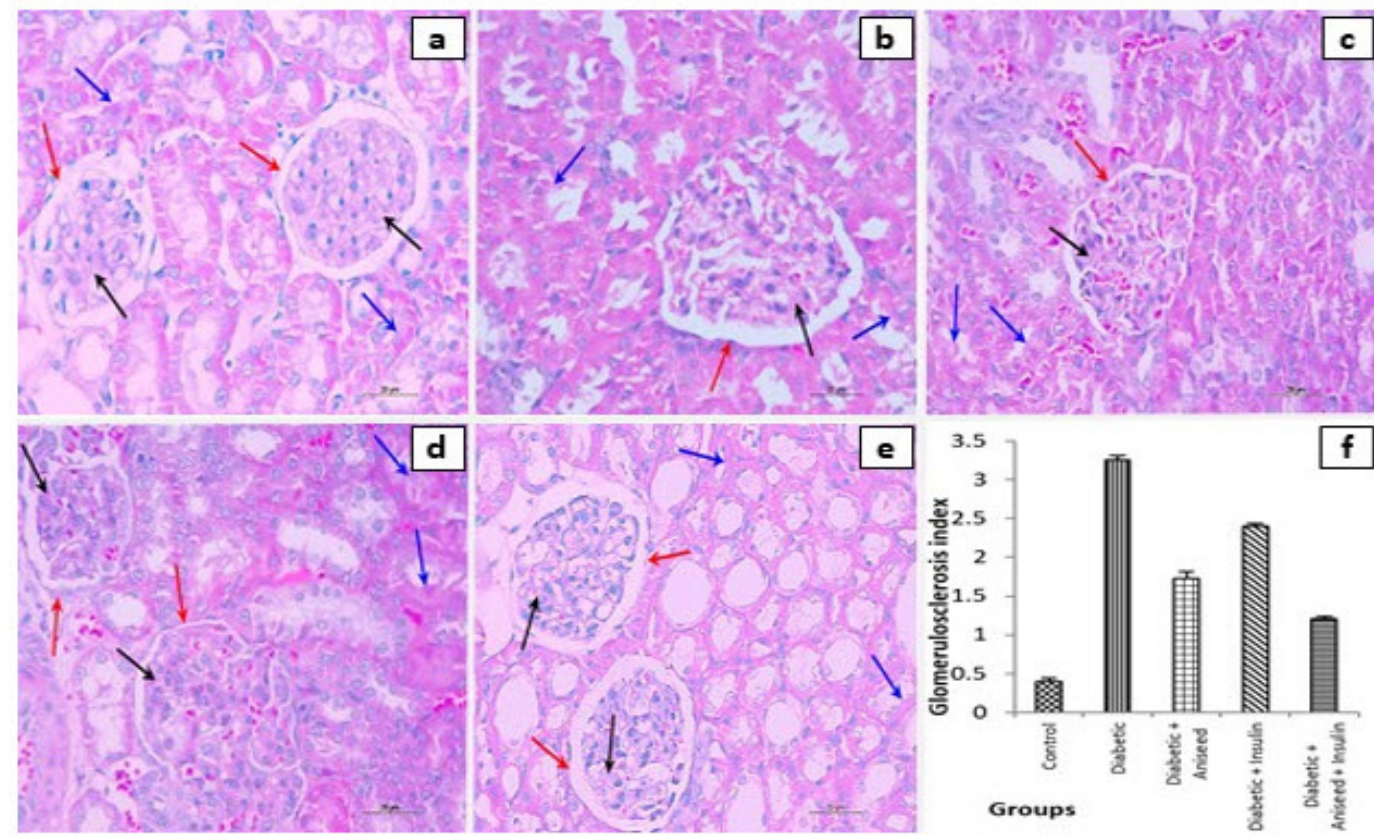

Fig. 4: Representative photomicrographs of the kidney from the group I (control): [a] PAS-positive material in the mesangium (black arrows), the basement membrane of the Bowman's capsule (red arrows), and the brush border of the PCTs (blue arrows) can be seen. group III (diabetic): [b] thickening basement membranes of the Bowman's capsule (red arrow), excess PAS positive material in the mesangium (black arrow), and the brush border of the PCTs (blue arrows) can be seen. Group IV (aniseed treated): [c] mild positive PAS reaction in the mesangium (black arrow), basement membrane of the Bowman's capsule (red arrow) and the brush border of the PCTs (blue arrows) can be seen. Group V (insulin-treated): [d] moderate positive PAS reaction in the mesangium (black arrows), basement membrane of the Bowman's capsule (red arrows), and the brush border of the PCTs (blue arrows) can be seen. Group VI (combined treated) [e] slight PAS positive material in the mesangium (black arrows), the basement membrane of the Bowman's capsule (red arrows), and the brush border of the PCTs (blue arrows) can be seen. [f] Graph of GSI. PAS [ a,b,c,d,e X 400, Scale bar: $20 \mu \mathrm{m}]$
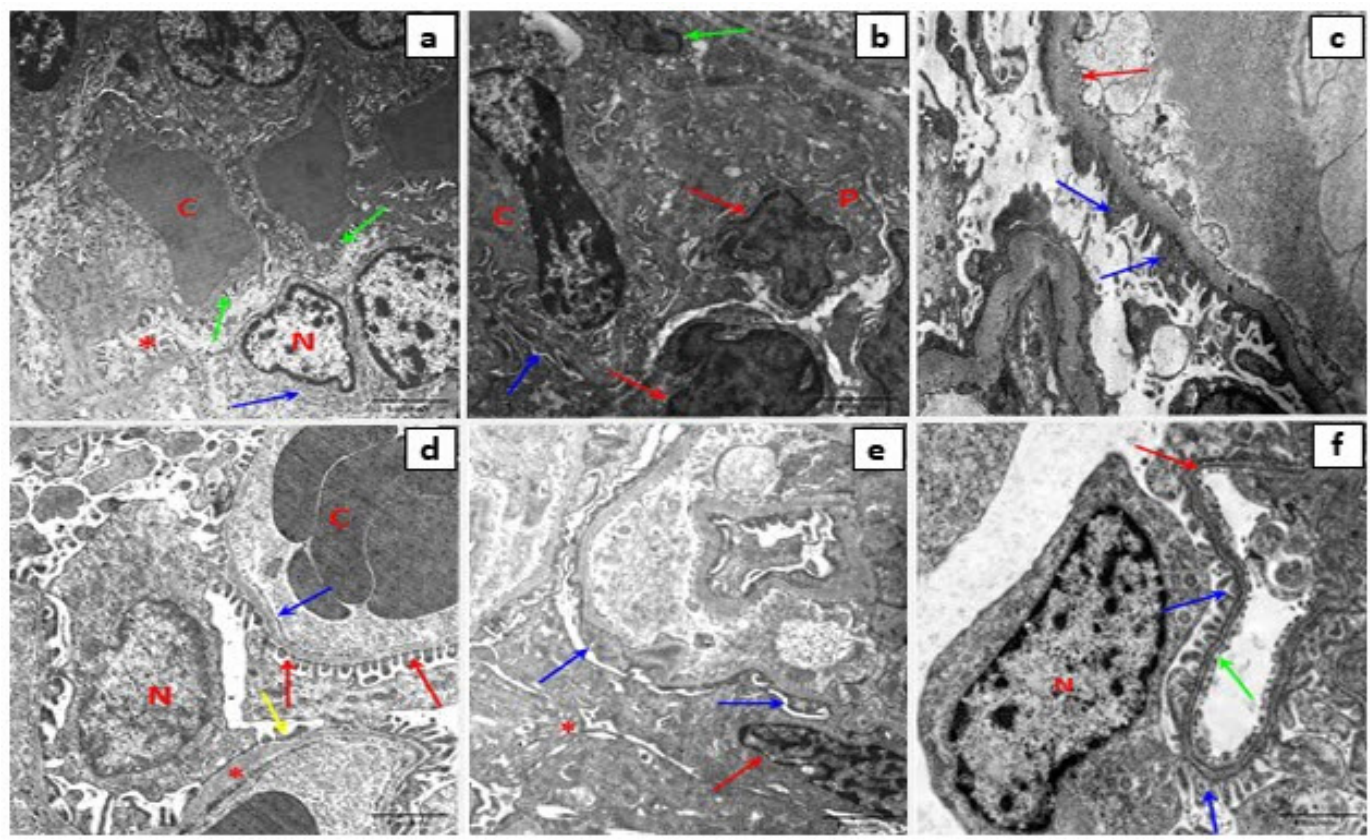

Fig. 5: Representative electron micrographs of the podocytes from Group I (control) [a] showing the podocytes (blue arrow) with their stellate or bizarre shaped nuclei $(\mathrm{N})$ wrapping around the blood capillaries $(\mathrm{C})$ and has several cytoplasmic extensions, primary processes (asterisk) and numerous secondary foot processes, pedicles (green arrows). Group III (diabetic): [b] podocytes (p) wrapping around congested glomerular capillary (C)with a fusion of secondary foot processes (blue arrow). hyperchromatic podocyte nuclei with peripheral condensation of chromatin (red arrows) and one is shrunken and pyknotic (green arrow) can also be seen. [c] a podocyte with a fusion of its secondary foot processes (blue arrows) with thickening of the glomerular capillary basal lamina (red arrow) can be noticed. Group IV (aniseed treated): [d] podocyte nucleus (N) wrapping around the blood capillaries (C) and its secondary foot processes (pedicles) having slit pores (red arrows) in between except with partial fusion of a few of them (yellow arrow) can be noticed. The pedicles envelop the capillary basement membrane (blue arrow) that showing partial thickening (asterisk). Group V (insulin-treated): [e] the podocyte with hyperchromatic nucleus (red arrow), diminution of their primary processes (asterisk), and fusion of secondary foot processes (blue arrows) can be seen. Group VI (combined treated): [f] the podocyte $(\mathrm{N})$, its secondary foot processes (pedicles) (blue arrows) and the pedicles envelop the capillary basement membrane (red arrow) appear normal with fenestrated endothelium (green arrow). TEM [(a, b,d,e,f) X 8000 c x17500 ] 


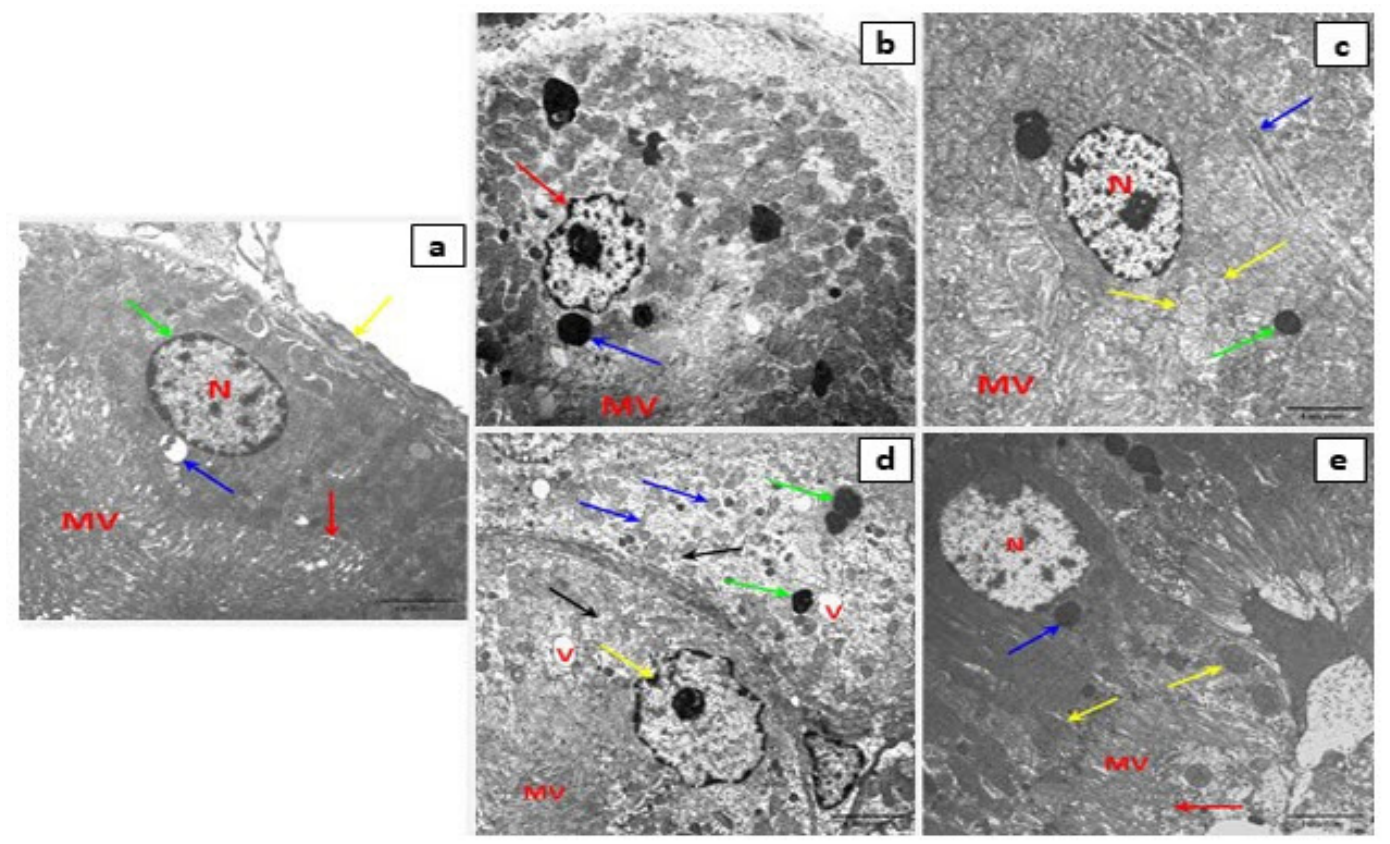

Fig. 6: Representative electron micrographs of the PCT from Group I (control) [a] showing PCT with a prominent continuous brush border (red arrow), intact basal striation (yellow arrow), and long closely packed apical microvilli (MV). A large oval euchromatic nucleus (N) with a regular nuclear membrane (green arrow) and pinocytotic vesicle (blue arrow) can be noticed. Group III (diabetic): [b] PCT appears with an abnormally shrunken nucleus, irregular nuclear envelop (red arrow) and microvilli (MV). Large-sized lysosomes (blue arrow) can be seen. Group IV (aniseed treated): [c] PCT with oval euchromatic nucleus $(\mathrm{N})$, intact basal striations (blue arrow), and apical microvilli (MV) projecting into the lumen, numerous mitochondria (yellow arrows) with few lysosomes (green arrow) can be noticed. Group V (insulin-treated): [d] PCT with irregular hyperchromatic nucleus (yellow arrow) and apical microvilli (MV) can be noticed. Most of the mitochondria appear normal (blue arrows), others degenerated (black arrows). Large-sized lysosomes (green arrows) and vacuolations (V) can be seen. Group VI (combined treated): [e] PCT appears with an oval euchromatic nucleus (N), a continuous brush border (red arrow), and normal mitochondria (yellow arrows). Microvilli (MV) projecting into the lumen and few lysosomes (blue arrow) can be noticed. TEM [(a, c,d,e) X $8000 \mathrm{~b} x 17500$ ]

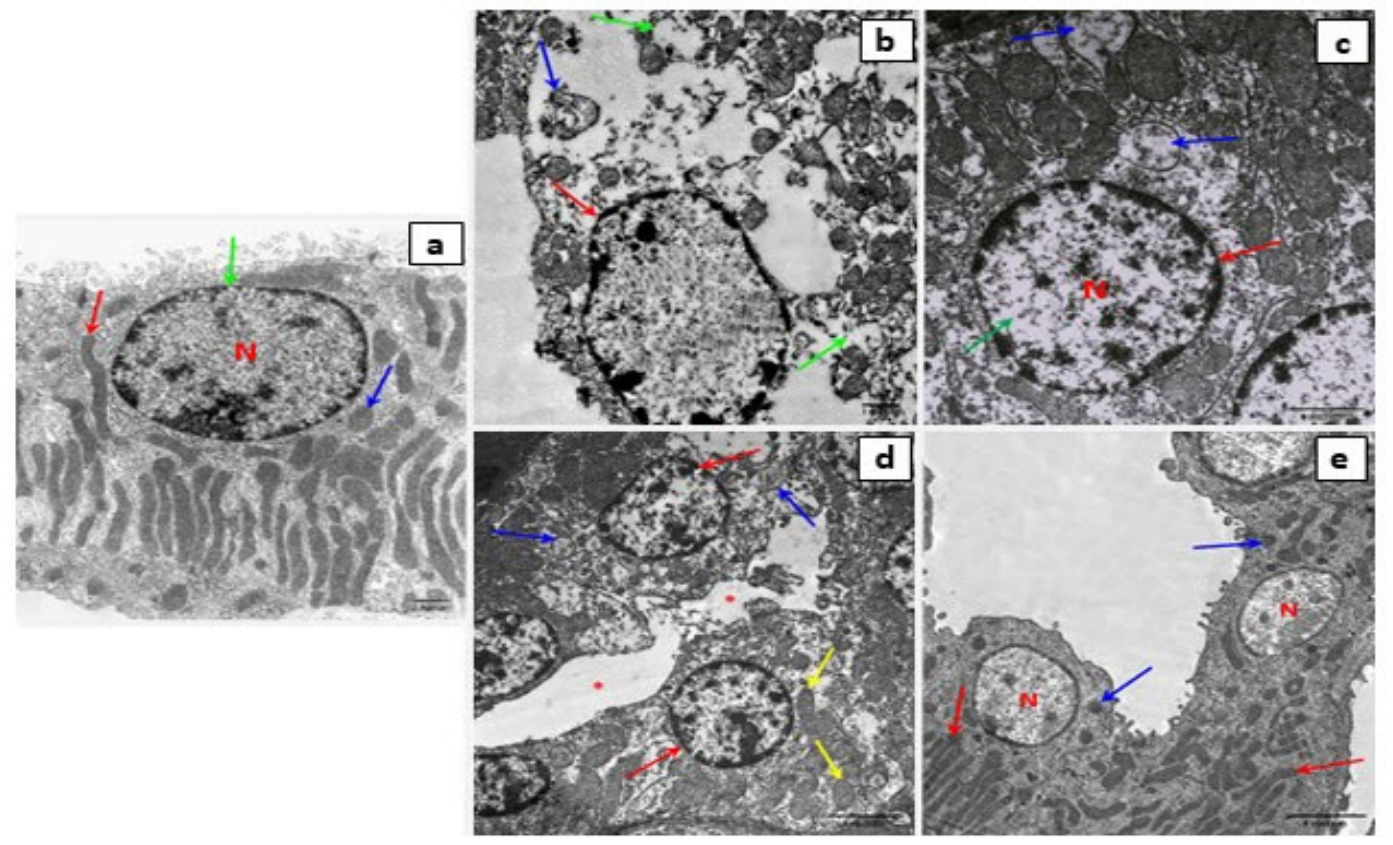

Fig. 7: Representative electron micrographs of the DCT from Group I (control) [a] showing DCT with apical euchromatic nucleus (N), regular nuclear membrane (green arrow), numerous elongated (red arrow) and rounded (blue arrow) mitochondria can be noticed. Group III (diabetic): [b] DCT appears with peripheral condensation of its chromatin (red arrow), destructed mitochondria (blue arrow) and rarefied its cytoplasm (green arrows). Group IV (aniseed treated): [c] DCT appears with oval \& euchromatic nucleus (N), regular nuclear envelop (red arrow), mild chromatolysis (green arrow), and massive mitochondrial lysis (blue arrows). Group V (insulin-treated): [d] DCTs appear with euchromatic nuclei (red arrows) and some mitochondria appear more or less normal (yellow arrows), others show loss of mitochondrial cisterns (blue arrows), and wide intercellular space (asterisks) can be noticed. Group VI (combined treated): [e] cells of DCT show normal euchromatic rounded to oval nuclei $(\mathrm{N})$ with elongated (red arrows) and rounded (blue arrows) mitochondria appear normal. TEM [(a, c,d,e) X 8000 b x17500 ] 


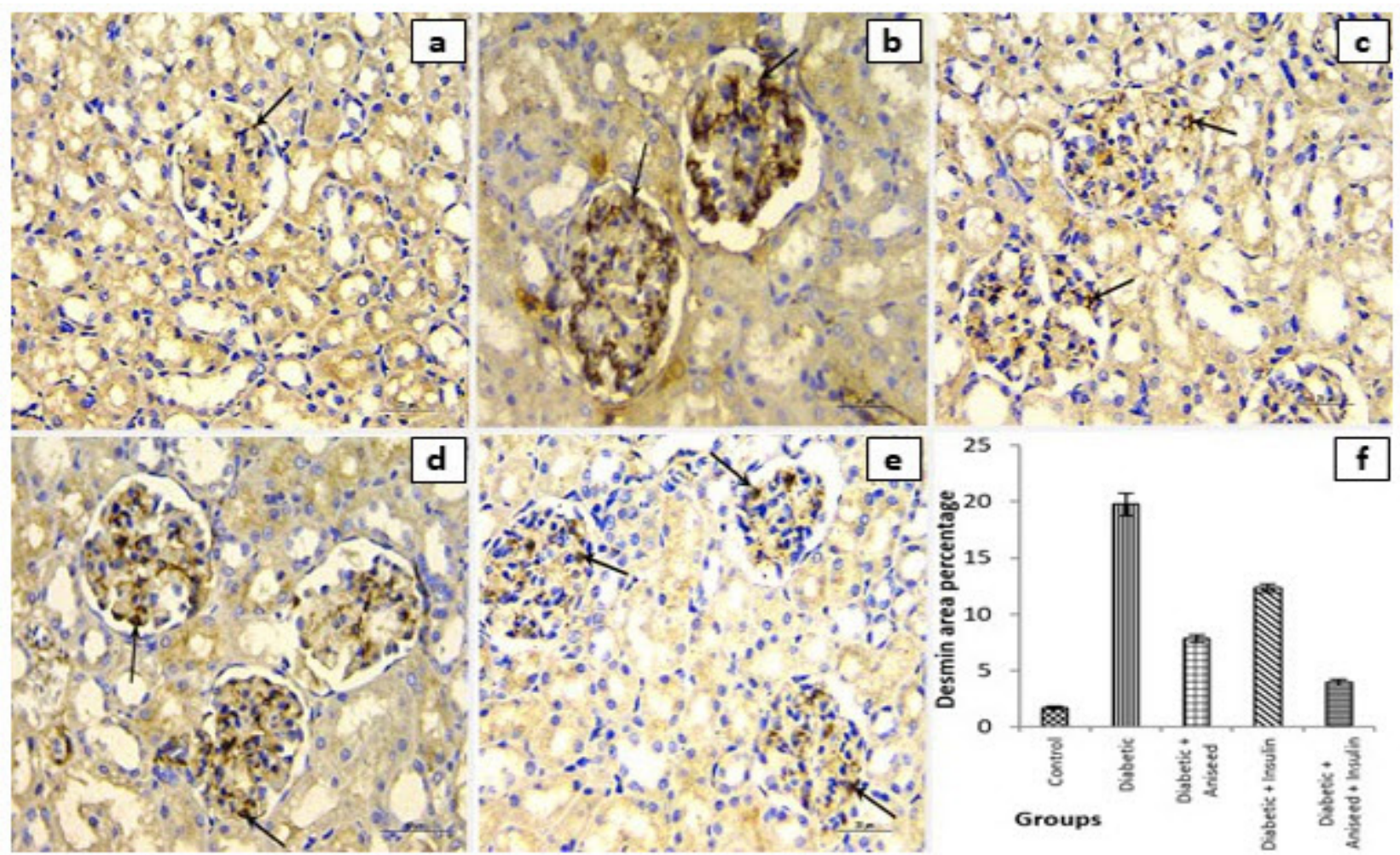

Fig. 8: Representative photomicrographs of the kidney from the group I (control): [a] and Group VI (combined treated): [e] slight positive immune-staining to desmin within the glomerulus can be noticed. Group III (diabetic): [b] strong positive immune-staining to desmin within the glomerulus can be seen. Group IV (aniseed treated): [c] mild immunoreaction to desmin within the glomerulus can be observed. Group V (insulin-treated): [d] moderate positive immunestaining to desmin within the glomerulus can be noticed (black arrows). [f] Graph showing desmin area percentage. Desmin [a,b,c,d,e X 400, Scale bar: $20 \mu \mathrm{m}$ ]
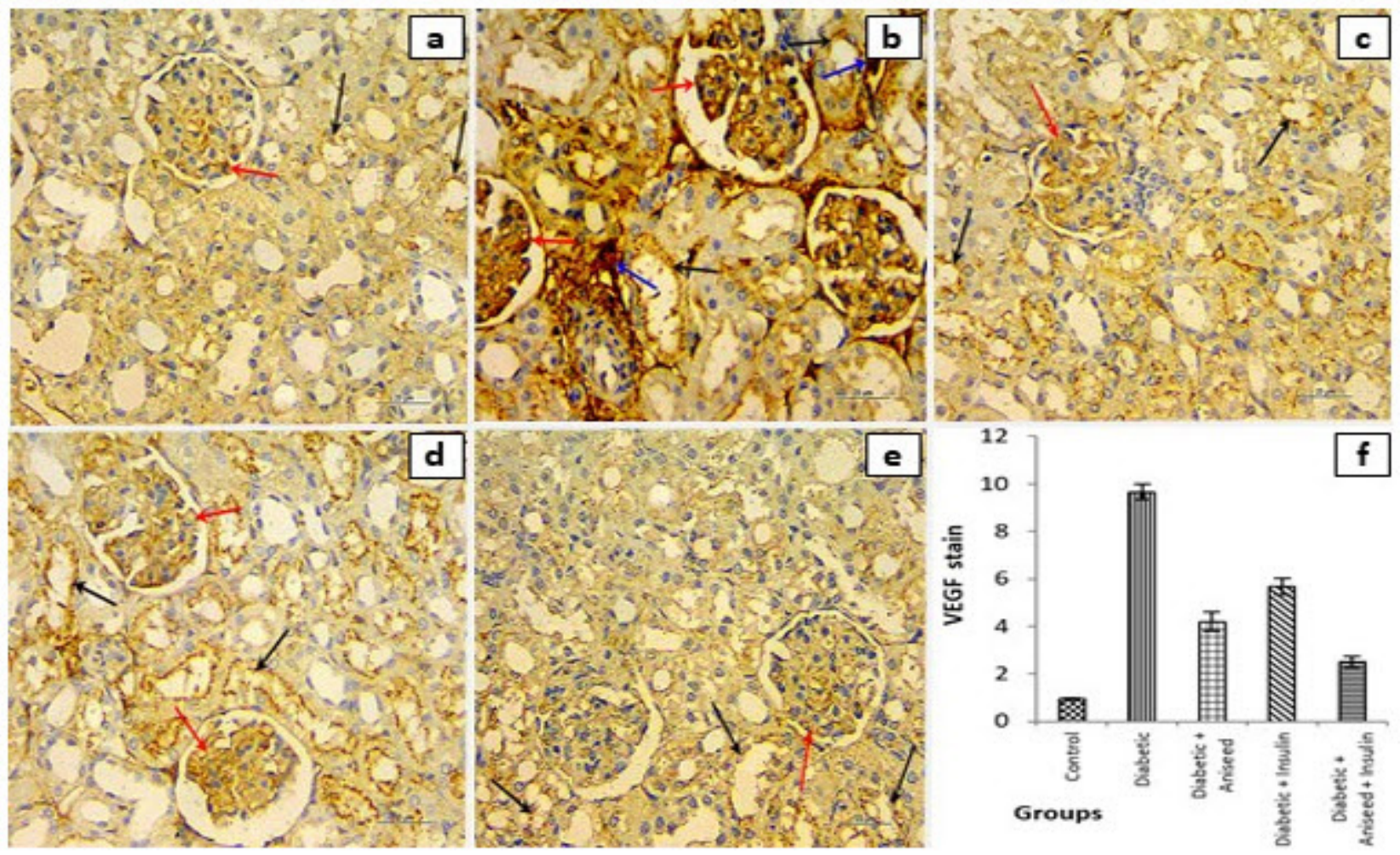

Fig. 9: Representative photomicrographs of the kidney from the group I (control): [a] and Group VI (combined treated): [e] slight cytoplasmic expression of VEGF in few renal tubules (black arrows) and in the glomerulus (red arrow) can be noticed. Group III (diabetic): [b] sever immunoreaction to VEGF within the glomerulus (red arrows), in the cytoplasm of the surrounding tubules (black arrows) and among the tubules (blue arrows) can be noticed. Group IV (aniseed treated): [c] mild cytoplasmic expression of VEGF in the renal tubules (black arrows) and in the glomerulus (red arrow) can be noticed. Group V (insulintreated): [d] moderate positive immunoreaction to VEGF in some renal tubules (black arrows) in the glomerulus (red arrows) can be noticed [f] Graph showing VEGF area percentage. VEGF [ a,b,c,d,e X 400, Scale bar: $20 \mu \mathrm{m}$ ] 

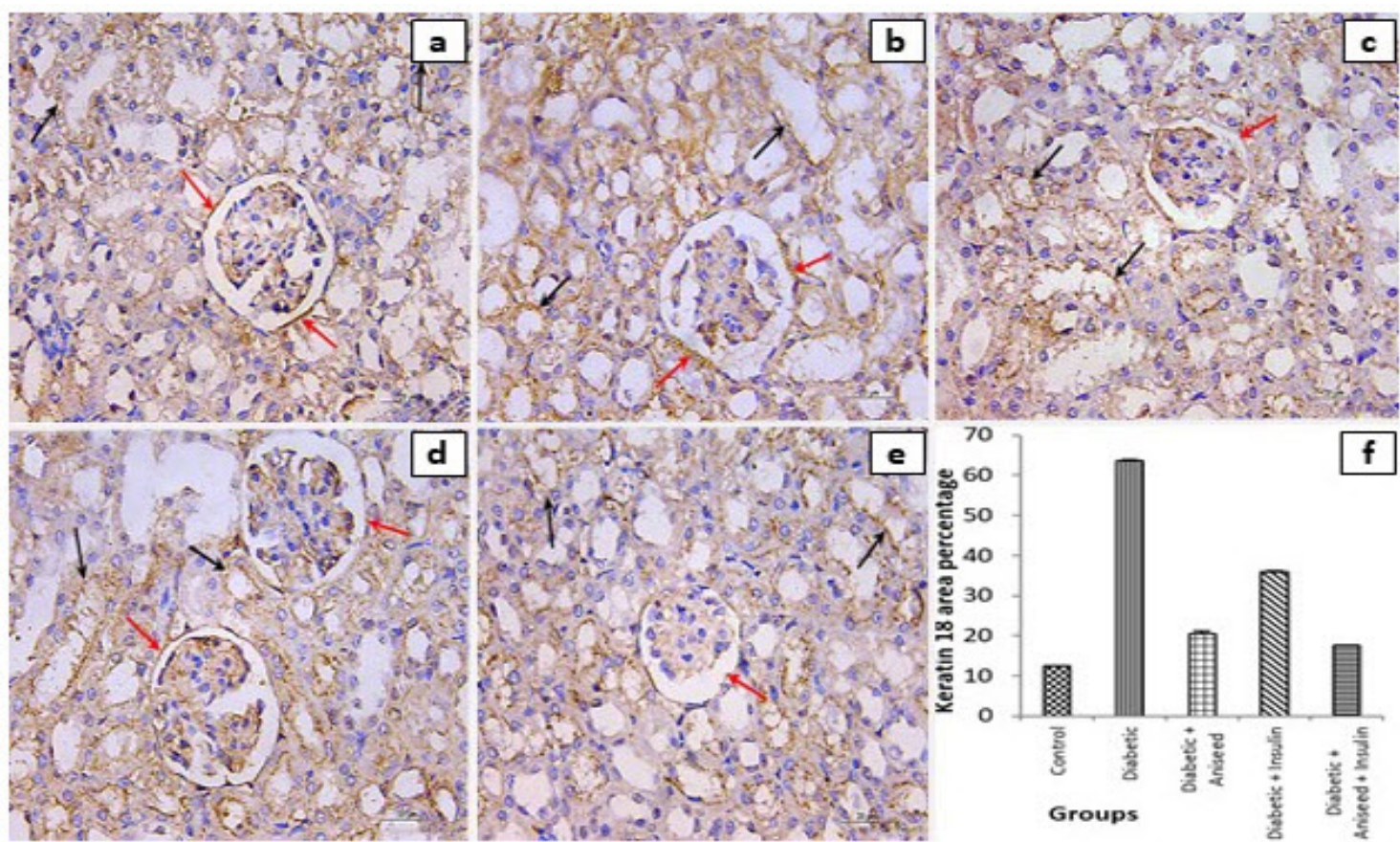

Fig. 10: Representative photomicrographs of the kidney from the group I (control): [a] and Group VI (combined treated): [e] show slight expression of keratin 18 in the parietal (red arrows) and renal tubular epithelial cells (black arrows). Group III (diabetic): [b] upregulation of keratin 18 in the parietal epithelial cells (red arrows) and renal tubular epithelial cells (black arrows) can be noticed. Group IV (aniseed treated): [c] down-regulation of keratin 18 in most of the parietal epithelial cells (red arrow) and renal tubular epithelial cells (black arrows) can be observed. Group V (insulin-treated): [d] moderate expression of keratin 18 in the parietal epithelial cells (red arrow) and the renal tubular epithelial cells (black arrows) can be noticed. [f] Graph showing keratin 18 area percentage. keratin 18 [ a,b,c,d,e X 400, Scale bar: $20 \mu \mathrm{m}$ ]

Table 1: showing mean \pm SD of body weight, blood glucose levels $(\mathrm{mg} / \mathrm{dl})$ and glycosylated hemoglobin (HbA1c) levels in all groups

\begin{tabular}{cccc}
\hline Groups & body weight (grams) & blood glucose levels (mg/dl) & (HbA1c) \\
\hline Group I & $245 \pm 18.51$ & $111.38 \pm 8.51$ & $4.41 \pm 0.34$ \\
Group III & $132.25 \pm 4.33^{* *}$ & $387 \pm 16.89^{*}$ & $12.76 \pm 0.37^{*}$ \\
Group IV & $186.25 \pm 29.73^{\circ}$ & $285.88 \pm 13.28^{\circ}$ & $9.40 \pm 0.39^{\circ}$ \\
Group V & $222.50 \pm 19.91^{\circ}$ & $242.75 \pm 9.72^{\circ}$ & $7.07 \pm 0.31^{\circ}$ \\
Group VI & $225.56 \pm 22.39 \star$ & $228.6 \pm 2.72^{\circ}$ & $6.05 \pm 0.17^{\circ}$ \\
\hline
\end{tabular}

** significant decrease compared with control group * significant increase compared to the control

- significant increase compared with diabetic group $\quad{ }^{\circ}$ significant decrease compared with diabetic group

Table 2: showing mean $\pm \mathrm{SD}$ of plasma urea $(\mathrm{mmol} / \mathrm{L})$ and plasma creatinine $(\mu \mathrm{mol} / \mathrm{L})$ levels in all groups

\begin{tabular}{ccc}
\hline Groups & plasma urea $(\mathrm{mmol} / \mathrm{L})$ & plasma creatinine $(\mu \mathrm{mol} / \mathrm{L})$ \\
\hline Group I & $27.37 \pm 1.30$ & $0.34 \pm 0.12$ \\
Group III & $66.50 \pm 1.51^{*}$ & $1.96 \pm 0.33^{*}$ \\
Group IV & $34.00 \pm 4.98^{\circ}$ & $0.54 \pm 0.08^{\circ}$ \\
Group V & $53.25 \pm 6.22^{\circ}$ & $0.81 \pm 0.15^{\circ}$ \\
Group VI & $28.5 \pm 2.67^{\circ}$ & $0.21 \pm 0.08^{\circ}$ \\
\hline
\end{tabular}

* significant increase in comparison with the control

${ }^{\mathrm{o}}$ significant decrease compared with the diabetic untreated

Table 3: showing mean \pm SD of SOD (U/mg) and MDA(Nmol/mg) and ACE-1 $(\mathrm{pg} / \mathrm{mg})$ levels in renal tissue in all groups

\begin{tabular}{cccc}
\hline Groups & SOD $(\mathrm{U} / \mathrm{mg})$ & MDA(Nmol/mg) & ACE-1(pg/mg) \\
\hline Group I & $2.4 \pm 0.63$ & $2.82 \pm 0.1$ & $2.04 \pm 0.32$ \\
Group III & $0.74 \pm 0.14^{*}$ & $9.3 \pm 0.38^{* *}$ & $0.48 \pm 0.76^{*}$ \\
Group IV & $1.7 \pm 0.09^{\circ}$ & $5.12 \pm 0.74^{*}$ & $1.41 \pm 0.16^{\circ}$ \\
Group V & $1.31 \pm 0.36^{\circ}$ & $6.2 \pm 0.74^{*}$ & $1.02 \pm 0.19^{\circ}$ \\
Group VI & $2.36 \pm 0.26^{\circ}$ & $3.5 \pm 0.73^{*}$ & $1.75 \pm 0.17^{\circ}$ \\
\hline
\end{tabular}

* significant decrease compared with the control

** significant increase in comparison with the control

${ }^{\circ}$ significant increase in comparison with the diabetic untreated significant decrease compared with the untreated diabetic 
Table 4: showing mean \pm SD of renal corpuscle diameter, area $\%$ of collagen fiber, GSI and median, range of score of the tubular injury in different groups

\begin{tabular}{|c|c|c|c|c|}
\hline Groups & renal corpuscle diameter & area $\%$ of collagen fiber & GSI & tubular injury score \\
\hline Group I & $121.17 \pm 3.49$ & $8.5 \pm 1.87$ & $0.4 \pm 0.089$ & $0.3(0-1)$ \\
\hline Group III & $89.5 \pm 6.66^{*}$ & $40.33 \pm 3.44^{* *}$ & $3.25 \pm 0.15^{* *}$ & $2.3(1-3)^{* * *}$ \\
\hline Group IV & $105.33 \pm 2.25^{\circ}$ & $17.08 \pm 0.58$ & $1.73 \pm 0.2^{*}$ & $1.17(1-2)^{\bullet}$ \\
\hline Group V & $99 \pm 0.63^{\circ}$ & $22.17 \pm 2.31^{\bullet}$ & $2.4 \pm 0.089^{\star}$ & $1.5(1-3)^{\star}$ \\
\hline Group VI & $109 \pm 1.26^{\circ}$ & $12.17 \pm 0.99^{\star}$ & $1.2 \pm 0.11^{*}$ & $0.8(0-2)^{\star}$ \\
\hline
\end{tabular}

* significant decrease compared with the control

** significant increase compared to the control

${ }^{\circ}$ significant increase in comparison with diabetic untreated

- significant decrease in comparison with diabetic untreated

Table 5: showing mean \pm SD of area \% of (Desmin, VEGF, Keratin 18) in different groups

\begin{tabular}{cccc}
\hline Groups & area \% of desmin & area \% of VEGF & area \% of keratin 18 \\
\hline Group I & $1.71 \pm 0.34$ & $0.935 \pm 0.06$ & $12.41 \pm 0.27$ \\
Group III & $19.75 \pm 2.36^{*}$ & $9.67 \pm 0.75^{*}$ & $63.61 \pm 0.87^{*}$ \\
Group IV & $7.83 \pm 0.75^{\circ}$ & $4.2 \pm 0.96^{\circ}$ & $20.58 \pm 1.18^{\circ}$ \\
Group V & $12.33 \pm 0.81^{\circ}$ & $5.67 \pm 0.82^{\circ}$ & $35.81 \pm 0.61^{\circ}$ \\
Group VI & $3.95 \pm 0.62^{\circ}$ & $2.5 \pm 0.55^{\circ}$ & $17.5 \pm 0.38^{\circ}$ \\
\hline
\end{tabular}

* significant increase in comparison with the control $\quad{ }^{\circ}$ significant decrease in comparison with diabetic group

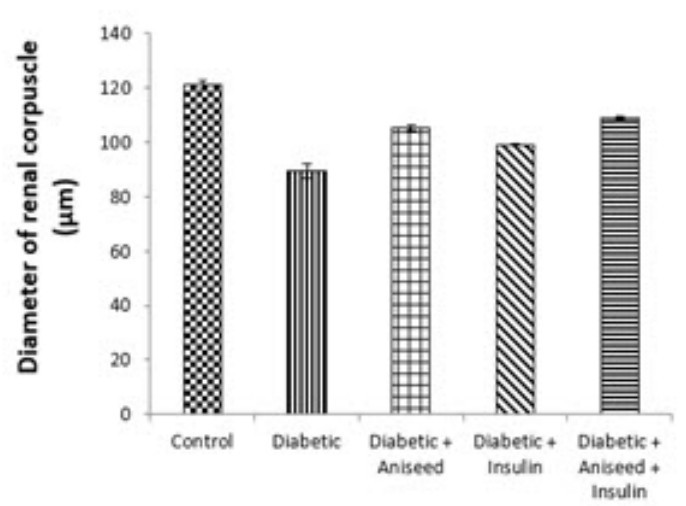

Graph 1: the mean of renal corpuscles diameter in all groups

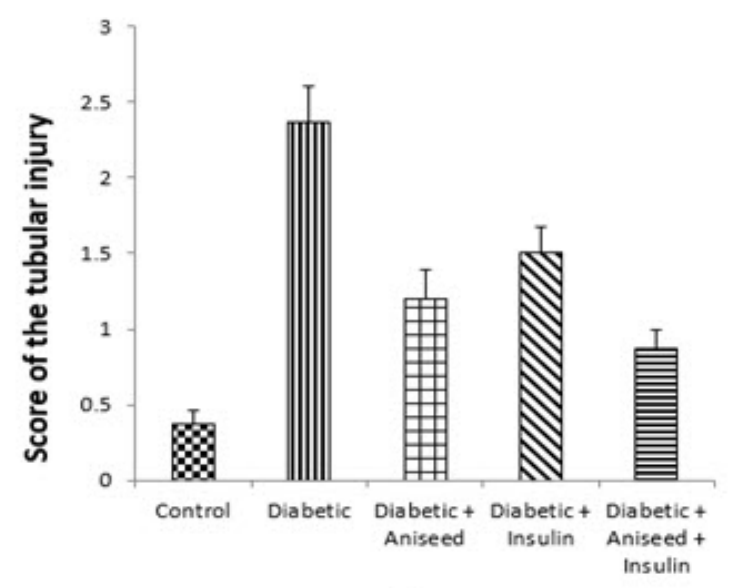

Graph 2: the mean of tubular damage score in all groups

\section{DISCUSSION}

Currently, Diabetes and its related complications such as diabetic nephropathy (DN) have ended up a major wellbeing issue ${ }^{[1]}$. long time of uncontrolled hyperglycemia leads to many organs failure with more death ${ }^{[14]}$. Diabetes and its complications affect body organs, especially the kidney ${ }^{[15]}$. In spite of the fact that a few drugs are utilized in an endeavor to control DM but culminate control is once in a while accomplish. Also, these drugs have numerous side impacts. So, searching for new medications with safe and effective properties in hyperglycemia control and delaying its complications is highly important ${ }^{[16]}$. Natural plants have been reported to possess myriad pharmacological activity with a great margin of safety; therefore, they are used in the treatment of diabetes and long-standing diabetic complications $^{[15]}$ Therefore, the point of our think about is to examine the role of aniseed extract in enhancing the biochemical and histological changes in diabetic nephropathy rat model and to compare its effect with insulin and with their combination.

Diabetic complications are caused by reactive oxygen species (ROS) that due to uncontrolled hyperglycemia. Then, ROS increases lipid peroxidation level that is the cause of diabetes complications ${ }^{[1]}$. Diabetic complications are also caused by deficiency in the body's antioxidant defense systems, damages to DNA, cellular membranes, and organelles $^{[2]}$. Also, free radical generation and exhaustion of antioxidant resistances creating a state of oxidative stress due to uncontrolled hyperglycemia that leads to more $\beta$-cells devastation and diabetic complications happens ${ }^{[16]}$. So, the dose of aniseed extract used in the study, $500 \mathrm{mg} / \mathrm{kg}$ was proved previously to have antioxidant properties ${ }^{[8]}$. 
The diabetic group showed a significant decrease in the final body weight, this was in accordance with ${ }^{[17]}$ who stated that STZ-induced DM is accompanied with a critical diminishment within the body weight due to high blood glucose level. Also, ${ }^{[9]}$ explained that wasting of protein and fatty acid stores caused by insulin deficiency could lead to weight loss due to the disability to metabolize carbohydrates which shifts fuel sources to fatty acids and proteins as energy sources.

In the diabetic group of the current study, there was a significant increase in their plasma urea and creatinine with major pathological lesions affecting both the glomeruli and the renal tubules which accompany hyperglycemia that occurs in diabetes. This was in accordance with ${ }^{[17]}$ who stated that elevated plasma urea and creatinine are the hallmark of DN. Also, there was a significant increase in the (HbAlc) percentage of the diabetic control group and this was in agreement with ${ }^{[18]}$ who stated that $\mathrm{HbAlc}$ is highly correlated with long-term hyperglycemia. Moreover, ${ }^{[19]}$ stated that $\mathrm{HbA1c}$ is an effective predictor of microvascular complications

Aniseed shown a significant hypoglycemic impact in comparison with the diabetic untreated. This was mostly related to its capacity to diminish the glycated hemoglobin level and ${ }^{[20]}$ expressed that a few phenolic compounds in anise meddled with the glucose absorption and this was agreed with ${ }^{[21]}$ who attributed its hypoglycemic effect to its antioxidant properties. Also, ${ }^{[22]}$ explained its antidiabetic activity due to tannins in addition to their $\alpha$-glucosidase inhibitory activity that inhibits insulin degradation and improves glucose utilization. This was in agreement with our study by significant reduction in malondialdehyde (MDA) level and an increase of superoxide dismutase (SOD) by aniseed extract and this was in line with ${ }^{[23]}$ who decided that anise increase insulin secretion from pancreatic $\beta$-cells in the diabetic neurotoxicity and so lowering blood glucose level.

In the current study, aniseed treated group showed a significant increase in the body weight in comparison with the diabetic untreated. This may be related to the hypoglycemic properties that avoiding hyperglycemiawhich is the cause of protein loss. Also, ${ }^{[24]}$ referred that to its dynamic compounds that have a stimulant impact on the digestive system. Moreover, aniseed provided a significant reduction in the plasma urea and creatinine and supported by histopathological evaluation. This was in agreement with ${ }^{[25]}$ who found that aniseed extract given marked renal protection against kidney damage by gentamicin in rats as evidenced by a significant diminishment in biochemical parameters.

In our insulin-treated group, insulin produced ameliorative effects on blood glucose level, body weight, renal tissue level of MDA and ACE, the activity of SOD, and histopathological alterations induced by STZ. This was in agreement with ${ }^{[9]}$ who indicated that insulin improved metabolic features as high blood glucose, glycated hemoglobin, and body weight loss and also restored some STZ-induced cardiomyopathy of diabetic rats. Although, glomerular basement membrane thickening and the expansion of mesangium decreased in insulin-treated group and this was in the line with ${ }^{[26]}$ and explained by ${ }^{[27]}$ that insulin deficiency activates and increases cell proliferation, fibronectin and collagen IV synthesis in renal mesangial cells which contributes to DN. Thus, early administration of insulin might prevent cell proliferation and so create a treatment target for DN.

Combined treatment with both anise and insulin produced a more significant recovering effect on renal tissue MDA level and SOD activity changes induced by STZ as compared to each agent alone. This was in agreement with ${ }^{[9]}$ who studied the combined treatment of crocin and insulin on diabetic heart tissue oxidative markers.

In the current study, the diabetic group showed a significant decrease in ACE levels. This was in agreement with $^{[28]}$ who stated that ACE is the key peptide of the reninangiotensin system and its plasma concentration increases but its kidney levels decreases of the diabetic rat model. Also, ${ }^{[29]}$ suggested that a low amount of ACE is harmful to the kidney in the case of prolonged hyperglycemia.

The distorted renal architecture was also proved in our study by TEM results that revealed destructed mitochondria, destructed microvilli, thickened glomerular capillary basement membrane, distorted minor processes, fused secondary foot processes, and peripheral condensation of the nuclear chromatin. This was supported by ${ }^{[1]}$ who explained these changes due to hyperglycemia and oxidative stress accompanied by DM. Also, the oxidative stress might clarify the significant increase in the MDA and decrease of SOD in the diabetic group.

Moreover, in our study, the diabetic group showed severe desmin immunoreaction that indicate the podocytes injury and this was in accordance with ${ }^{[30]}$ who found a significant increase in desmin protein in the kidneys of diabetic rats and referred these changes to the podocyte injury. The renal tubules demonstrated dilated congested blood vessels, vacuolations, desquamation, degenerated mitochondria, pyknotic nuclei, and wide intercellular spaces. This was in agreement with $^{[31]}$ who stated that the mitochondrial degeneration is due to the uncontrolled hyperglycemia that overpowers the electron transport chain of the mitochondria by increasing ROS generation and the oxidative phosphorylation. Furthermore, ${ }^{[32]}$ stated that high blood glucose level directly mediates apoptosis which clarifies the condensation of the nuclear chromatin and pyknotic nuclei in the present study.

In the present work, the diabetic group showed that the Bowman's space was either dilated or obliterated; this indicated that the disease in early and late stages respectively ${ }^{[33]}$. Dilatation of the capsular space might be due to shrinkage of the glomerular capillary tuft. Obliteration of the space could be due to mesangial cell expansion with the following sclerosis and this was in agreement with ${ }^{[33]}$ and this was confirmed in this study morphometrically that renal corpuscle diameters in the diabetic group were significantly diminished showing the event of shrinkage of the glomerular capillary tuft and corpuscular space dilatation. 
In this study, the diabetic group showed a significant increase in the deposition of collagen fiber. chronic inflammation might be the cause of the fibrosis, so prolonged exposure of fibroblasts to inflammatory mediators resulting in unusual collagen production. Also, there was a significant increase in GSI and glomerular basement membrane thickening and this was in agreement with ${ }^{[34]}$ who stated that $\mathrm{DN}$ is characterized by extracellular matrix accumulation and glomerular hypertrophy that leads to fibrosis of the renal tissue.

The diabetic group showed a highly significant upregulation in the keratin $18 \%$ surface area; this was in line with $^{[35]}$ and showed strong positive VEGF immunostaining; which is an index of reaching the angiogenesis; this was in accordance with ${ }^{[36]}$ who stated that oxidative stress activated angiogenesis pathway.

In our work, the anise treated group showed wonderful regenerative changes demonstrated by significant improvement in SOD and MDA levels and the notable amelioration of the distorted renal structure compared to the diabetic group. The diabetic insulin-treated group showed mild impressive improvement compared with the diabetic group. The present study showed a significant reduction in collagen fiber deposition of the anise treated group when compared to the insulin-treated one. This might suggest the anti-inflammatory effect of anise. So, regression of fibrosis could be due to regression of inflammation. This was in line with $^{[21]}$ who reported that anise might cause inhibition of transforming growth factor-beta 1 (TGF- $\beta 1)$. Also, ${ }^{[36]}$ studied the effect of anise in suppressing TGF- $\beta 1$ secretion in the lymphocytes and so had the ability of significantly delay the renal tissue fibrosis, that demonstrate the anti-fibrotic effect of anise. In the present study, anise treated diabetic group showed mild positive immunoreaction for desmin and keratin 18 which might prove the inhibition of renal cell injury and this finding was in agreement with ${ }^{[37]}$. Also, there was a decrease in VEGF expression and this was in agreement with $^{[38]}$ who found that anise repressed tumor angiogenesis inducer in vivo via a diminishing of angiogenic factors in neoplasms.

Furthermore, the diabetic control group showed increased tubular injury score due to vacuolations, desquamation, and dilatation of both PCTs and DCTs. SO, the renal parameters showed moderate improvement in the aniseed treated group, mild in the insulin-treated diabetic group, and marked amelioration in the anise plus insulin group and this is in agreement with ${ }^{[39]}$ who found the combination of insulin and metformin produces the best way in glucose control and diminished the renal damage by STZ induced gestational diabetes.

In this study, up-regulation of keratin 18 in the diabetic group was in line with ${ }^{[40]}$ who stated that keratin 18 is overexpressed within the kidney tissues of diabetic rat and added that in the adenine nephropathy model keratins were already highly overexpressed. Thus, keratins might serve as early and a good markers of tubular epithelial cell injury. With the progression of the renal injury by the disease, expression of keratins then increased. This proposes that the degree of keratin immunoreaction gets with the degree of damage. Also, ${ }^{[41]}$ stated that in Balkan endemic nephropathy, keratin 18 was up regulated in the tubular epithelium, mostly on the damaged proximal tubules. Confirmatory to our results, ${ }^{[42]}$ found an increase total serum keratin 18 concentration and was also highly increased in the urine in patients with late stages of chronic kidney disease than healthy patients.

The diabetic anise treated group presented with a significant improvement of the kidney architecture with a significant decrease in blood urea and creatinine as compared to the diabetic. The insulin diabetic treated group showed improvement in blood glucose level, some improvement in the SOD and MDA in the kidney tissue was observed, and mild improved structural renal lesions. This was in agreement with $^{[43]}$ who explained as the exogenous insulin directly acts on insulin receptors and opens the glucose transporter gates in the skeletal muscles and adipose tissues to allow entry of glucose inside the cells and improve hyperglycemia.

In our research, the diabetic aniseed treated group showed a diminishing of renal markers levels that can be an effective predicator in lowering of diabetic renal damages. This was in accordance with ${ }^{[2]}$ who stated that aniseed can prevent kidney damage in lead toxicity by lowering the level of lipid peroxidation due to the ability of anise in scavenging of free radicals. This was in agreement with ${ }^{[25]}$ who explained the improving of the architecture of glomerulus and renal tubules in anise treated group gives strong evidence for renoprotective role and with ${ }^{[44]}$ who examined its antioxidant activity in the prevention and treatment of liver injury in rats.

Insulin is the most effective glucose-lowering agent among all diabetes drugs. It can reduce any level of hyperglycemia to target levels and is easy to avoid hypoglycemia due to delayed its clearance by the kidney ${ }^{[45]}$. In comparison with aniseed alone, insulin treatment alone provided better control of body loss and hyperglycemia induced by STZ, but partially improved STZ-induced biochemical and histopathological changes and this is due to the dual actions of anise (antioxidant and anti-diabetic effects) and this is in agreement with ${ }^{[21]}$.

Thus, aniseeds are the favorable source of nutraceuticals which are effective protective agents against stressinduced diseases such as diabetes. our findings have also demonstrated that treatment with aniseed plus insulin has further advantages to adjustment of metabolic and renal tissue biochemical and histological alterations induced by STZ. It has been reported that a combination of insulin and spironolactone provided more benefits than the use of either agent alone in the treatment of general characteristics of diabetes-induced renal injury ${ }^{[43]}$. Others ${ }^{[9]}$ added that the use of combination therapy (insulin plus crocin) appears to have potential advantages especially in the reduction of oxidative stress in improving diabetic cardiomyopathy than one agent. 


\section{CONCLUSION AND RECOMMENDATION}

Our findings have shown that treatment with anise has a valuable effect on renal injuries caused by DM and aniseed plus insulin has more benefits to the normalization of biochemical and histological alterations induced by STZ. Up to our information, there are no recorded investigates showing the useful impact of treatment with aniseed plus insulin on STZ-induced DN.

So, advance considers are required to toss more light on the consider of anise impact on diverse diabetic complications and to look at the impact of distinctive dosages of anise extract on DM

\section{CONFLICT OF INTERESTS}

There are no conflicts of interest.

\section{REFERENCES}

1. Mestry S, Dhodi J, Kumbhar S, Juvekar, A. Attenuation of diabetic nephropathy in streptozotocin-induced diabetic rats by Punica granatum Linn. Leaves extract Journal of traditional and complementary medicine. 2017; 7(6): 273-280.

2. Fonseca C, Fernandes S, Cordeiro P, Watanabe F, Vattimo F. The role of oxidative stress in streptozotocininduced diabetic nephropathy in rats. Arch Endocrinol Metab.2016; 60(5): 443-449

3. Zhang X, Kong J, and Yun K. Prevalence of Diabetic Nephropathy among Patients with Type 2 Diabetes Mellitus in China: A Meta-Analysis of Observational Studies. Journal of Diabetes Research.2020; 1(1):2-14.

4. Gonzales ML, Thomas DB, Barisoni L, and Fornoni A. Diabetic nephropathy: Is it time yet for routine kidney biopsy? World J Diabetes. 2013;4(6):245-255.

5. Ahad A, Mujeeb M, Ahsan H and Siddiqui A. W. Nephroprotective potential of Quercus infectoria galls against experimentally induced diabetic nephropathy in rats through inhibition of renal oxidative stress and TGF- $\beta$, Animal Cells and Systems. 201620 (4): 193-202.

6. Yu TY, Wei JN, Kuo CH, Liou JM, Lin MS, Shih SR, Hua CH, Hsein YC, Hsu YW, Chuang LM, Lee MK, Hsiao $\mathrm{CH}, \mathrm{Wu} \mathrm{MS}, \mathrm{Li} \mathrm{HY}$. The Impact of Gastric Atrophy on the Incidence of Diabetes. Sci Rep. 2017;3(7):39777.

7. Koriem K. Approach to pharmacological and clinical applications of Anisi aetheroleum. Asian Pac J Trop Biomed. 2015; 5 (1): 60-67.

8. Ashtiyani CS, Seddigh A, Najafi A, Hossaini N, Avan A, Akbary A, Manian M, and Nedaeinia R. Pimpinella anisum L. ethanolic extract ameliorates the gentamicin induced nephrotoxicity in rats. Nephrology. 2017;22:133-138.

9. Farshid A, Tamaddonfard E, and Arzeloo M. The effects of crocin, insulin and their coadministration on the heart function and pathology in streptozotocin-induced diabetic rats. Avicenna J Phytomed. 2016; 6(6): 658-670.

10. Zangiabadi N, Sheibani V, Asadi-Shekaari M, Shabani M, Jafari M, Asadi AR, Tajadini H, and Jarahi M. Effects of Melatonin in Prevention of Neuropathyin STZ-Induced Diabetic Rats. American Journal of Pharmacology and Toxicology. 2011; 6 (2): 59-67.

11. Rezk H, El-Sherbiny M, Atef H, and Taha M. Effect of spironolactone on diabetic nephropathy in albino rats: ultrastructural and immunohistochemical study. International Journal of Scientific Reports. 2017; 3(5): 110-119

12. Zhang S, Xu H, Yu X, Wu Y, and Sui D. Metformin ameliorates diabetic nephropathy in a rat model of lowdose streptozotocin-induced diabetes. Exp Ther Med. 2017;14(1):383-390

13. Chen J, Ren J, WTY L, and Hao L, Lysyl oxidases expression and histopathological changes of the diabetic rat nephron. Mol Med Rep.2018;17(2):2431-2441

14. Al Trad B, Ashankyty I and Alaraj M. Progesterone ameliorates diabetic nephropathy in streptozotocin induced diabetic Rats. Diabetol Metab Syndr 2015; 5 (1):7-97.

15. Yi JK, Ryoo ZY, Ha JJ, Oh DY, Kim MO and Kim SH. Beneficial effects of 6-shogaol on hyperglycemia,islet morphology and apoptosis in some tissues of streptozotocin-induced diabetic mice. Diabetol Metab Syndr. 2019; 12; 11:15.

16. Jain D, and Saha S. Antioxidant and antihyperglycaemic effects of naringenin arrest the progression of diabetic nephropathy in diabetic rats. Egyptian Pharmaceutical Journal. 2017; 16:144-151.

17. Konda PY, Dasari S, Konanki S, and Nagarajan P. In vivo antihyperglycemic, antihyperlipidemic, antioxidative stress and antioxidant potential activities of Syzygium paniculatum Gaertn. in Streptozotocin-induced diabetic rats. Heliyon. 2019; 5(3):1373- 1384.

18. Goswami I, Mazumdar I, Sridhar K, and Narayanan M. Correlation of HbA1C levels with average Estimated blood Glucose levels in improvement of Diabetes Management. International Journal of Biotechnology and Biochemistry. 2019; 13(3):205-210

19. Juarez DT, Demaris KM, Goo R, Mnatzaganian CL, and Wong H. Significance of HbAlc and its measurement in the diagnosis of diabetes mellitus: US experience. Diabetes Metab Syndr Obes.2014; 7:487-494

20. Sun W, Shahrajabian MH, and Cheng Q. Anise (Pimpinella anisum L.), a dominant spice and traditional medicinal herb for both food and medicinal purposes, Cogent Biology.2019; 5(1):673-688. 
21. Shobha R, and Andallu B. Antioxidant, Anti-Diabetic and Hypolipidemic Effects of Aniseeds (Pimpinella anisum L.): In vitro and in vivo Studies. J Complement Med Alt Healthcare.2018; 5(2): 555-565

22. Shojaii, A. and Fard, AM. Review of Pharmacological Properties and Chemical Constituents of Pimpinella anisum. International Scholarly Research Network. 2012; (1):1-9.

23. EL-hodairy FA. Neuroprotective effects of pimpinella anisum on neurotoxicity full proceeding paper induced by bisphenol on normal and diabetic rats. International Journal of Pharmacy and Pharmaceutical Sciences. 2014; 6(3) 54-61.

24. Bekara A, Ait HA, Kahloulakhaled SN, and Aoues A. Nephroprotective effect of Pimpinella anisum L. aqueous extract against lead toxicity: In vivo study. International Journal of Green Pharmacy. 2016;10 (2): 86-90.

25. AiswaryaN,RashmiR,PreethiJS,ChandranV,Teerthanath S, Sunil PB, and Rakesh KB. Nephroprotective Effect of Aqueous Extract of Pimpinella anisum in Gentamicin Induced Nephrotoxic $\neg$ ity in Wistar Rats. Pharmacog J. $2018 ; 10(3): 403-407$.

26. Michael ST, Ganesh RN, and Viswanathan P. Effect of long acting insulin supplementation on diabetic nephropathy in Wistar rats. Indian $\mathrm{J}$ Exp Biol. 2012; 50(12):867-874

27. Gong D, Wang L, Yang Y, Zhang J, and Liu X, Diabetes aggravates renal ischemia and reperfusion injury in rats by exacerbating oxidative stress, inflammation and apoptosis. Renal Failure.2019; 41(1):750-761

28. Al-Qattan KK; Thomson M, Jayasree D, and Ali M. Garlic Attenuates Plasma and Kidney ACE-1 and Ang II Modulations in Early Streptozotocin-Induced Diabetic Rats: Renal Clearance and Blood Pressure Implications. Evidence-Based Complementary and Alternative Medicine.2016; 3(2): 2-10.

29. Bertoncello N, Moreira R, Arita d, Aragao D, Dantas P, Santos R, and Yokota R. Diabetic Nephropathy Induced by Increased Ace Gene Dosage Is Associated with High Renal Levels of Angiotensin (1-7) and Bradykinin. Journal of Diabetes Research 2015; 10 (2):401-407.

30. Xu H, Wang X, Liu, M. and He, X. Tangzhiqing Granules Alleviate Podocyte Epithelial-Mesenchym al Transition in Kidney of Diabetic Rats. Evidence-Based Complementary and Alternative Medicine. 2017; 12(1):1-9

31. Zhao RZ, Jiang S, Zhang L, and Yu ZB. Mitochondrial electron transport chain, ROS generation and uncoupling (Review). Int J Mol Med.2019; 44(1):3-15.

32. Weil EJ, Lemley KV, Yee B, Lovato T, Richardson M, Myers BD, and Nelson, RG. Podocyte detachment in type 2 diabetic nephropathy. Am J Nephrol.2011;1:21-24.
33. Mott A, Gasim A, Schober F, Hu Y, and Jennette J. (2018): Segmental Sclerosis and Extracapillary Hypercellularity Predict Diabetic. ESRDJ Am Soc Nephrol.2018; 29: 694-703.

34. Li, Z. and Zhang, W. Protective effect of berberine on renal fibrosis caused by diabetic nephropathy. Molecular Medicine Reports.2017;16: 1055-1062.

35. Eichinger LD, Krenn CG, and Roth G.A. Keratin 18 and heat-shock protein in chronic kidney disease. Adv.Clin. Chem.2013; 62:123-149

36. Sangwan P, and Khanna K. Consistency of Rat model for diabetic nephropathy, International Journal of Applied Research 2015; 1(9):157-159.

37. Rebey IB, Wannesa WA, and Kaaba S. Bioactive compounds and antioxidant activity of Pimpinella anisum L. accessions at different ripening stages. Scientia Horticulture.2019; 246:453-461.

38. $\mathrm{Kim} \mathrm{A}, \mathrm{Im} \mathrm{M}$, and Ma JY. Anisi stellati fructus extract attenuates the in vitro and in vivo metastatic and angiogenic potential of malignant cancer cells by down regulating proteolytic activity and pro-angiogenic factors. Int J Oncol.2014; 45(5):1937-1948.

39. Kassab BM, Hussein HH, Mahmoud OM, and Abdel-Alrahman G. Effects of insulin and metformin on fetal kidney development of streptozotocin-induced gestational diabetic albino rats. Anat Cell Biol. 2019; 52(2):161-175

40. Djudjaj S, Papasotiriou M, Roman D and Wagnerova A. Lindenmeyer, M.; Cohen, C. and Dimitrios, S. Keratins are novel markers of renal epithelial cell injury. Kidney International.2016; 89:792-808

41. Stefanovic V, Cukuranovic R, Dojcinov D, and Savic V. Coexpression of Vimentin and Cytokeratin in Damaged Tubular Epithelia of Kidney in Balkan Nephropathy. Nephron.1996; (72):119-120.

42. Roth GA, Lebherz-Eichinger D, and Ankersmit HJ. Increased total cytokeratin-18 serum and urine levels in chronic kidney disease. Clin Chim Acta. $2011 ; 412: 713-717$

43. Jiang Y, Zhang W, Xu S, Lin H, Sui W, Liu H, Peng $\mathrm{L}$, and Jinning J. Transplantation of human fetal pancreatic progenitor cells ameliorates renal injury in streptozotocin-induced diabetic nephropathy. J Transl Med.2017; 1-13.

44. Cengiza N, Ozbekb H, and Him A. Hepatoprotective effects of Pimpinella anisum seed extract in rats. Pharmacology online. 2008; 3:870-874.

45. Akbas F. Protective effect of insulin treatment on early renal changes in streptozotocin induced diabetic rats Acta Endocrinol (Buchar). 2018;14(2):169-174 


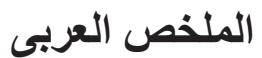

\section{التأثير الوقائي لليانسون، الانسولين وكليهما معاً على إعتلال الكلية فى نموذج مرض السكري في الفئران}

\section{رشا رجب سليمه، مصطفى محمود الحبيبي، عبير السيد الميهى، منار على فريا}

\section{قسم التشريح الآدمى كلية الطب - جامعة المنوفية}

المقدمة: يعد إعتلال الكلية من أكثر المشاكل المصاحبة لمرض السكرى فى جميع أنحاء العالم وقد يؤدى إلى الغسيل الكلوى أو زرع كلى جديدة.و على الرغم من العديد من الأبحاث التى أجريت على الأنسان أو الحيو انات إلا أنه لم يتم

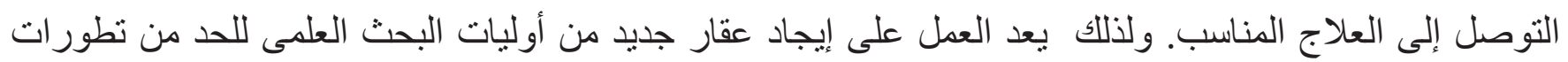
مرض السكرى .

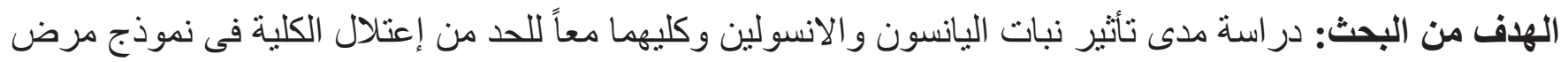

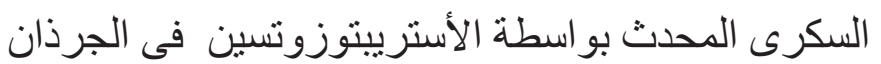
المواد والطرق: قسمت ست وخمسون من الجرذان المهق البالغة إلى ست مجموعات.المجموعة الآولى )الضابطة)

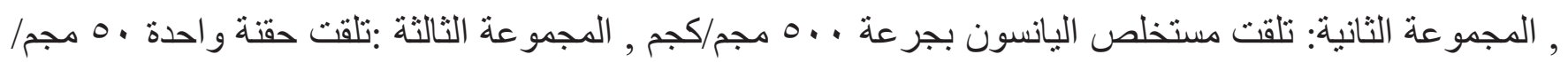
كجم أستربيتوزوسين بالحقن البرينونى, المجمو عة الر ابعة: بعد التأكد من الآصابة بمرض السكرى تم إعطاء الجرذان

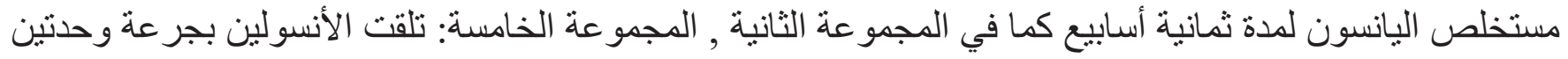

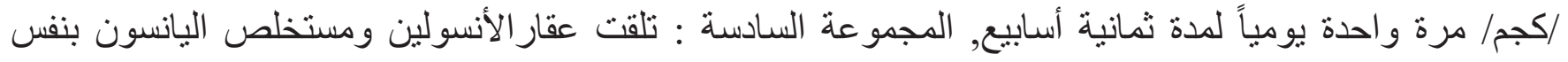
الجر عات السابقة لمدة ثمانية أسابيع. وفى نهاية التجربة تم تجهيز العينات لدر استها بالمحهر الضوئئى و الألكترونى النافذ. كما أجريت لها در اسات قياسية و إحصائية النتائج: أظهرت الجرذان المصابة بمرض السكرى اضطر اب في التركيب النسيجى لكلا من كريات الكلية والأنابيب

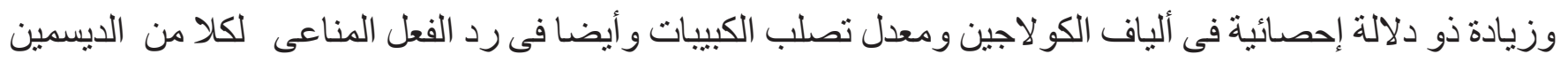

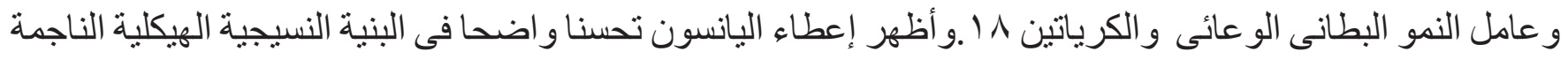

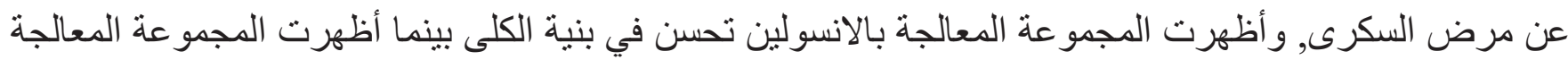

باليانسون و الانسولين معا تغيير ات مؤثرة وو اعدة فى الحد من الإجهاد التأكسدى فى تحسين إعتلال الكلية السكرى

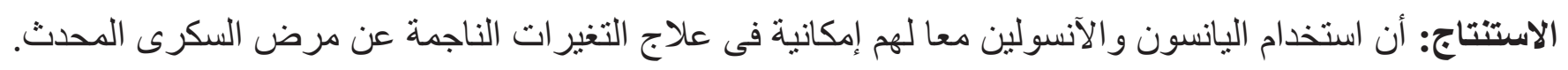

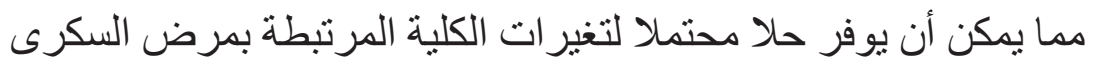

